\title{
Investigation on dynamics of an impulsive predator-prey system with generalized Holling type IV functional response and anti-predator behavior
}

\author{
Sekson Sirisubtawee ${ }^{1,2^{*}}$ (D) Nattawut Khansai ${ }^{1}$ (D) and Akapak Charoenloedmongkhon ${ }^{1,2}$ (B)
}

"Correspondence:

sekson.s@sci.kmutnb.ac.th

1 Department of Mathematics,

Faculty of Applied Science, King

Mongkut's University of Technology

North Bangkok, 10800 Bangkok,

Thailand

${ }^{2}$ Centre of Excellence in

Mathematics, CHE, Si Ayutthaya

Road, 10400 Bangkok, Thailand

\section{Springer}

\begin{abstract}
In the present article, we propose and analyze a new mathematical model for a predator-prey system including the following terms: a Monod-Haldane functional response (a generalized Holling type IV), a term describing the anti-predator behavior of prey populations and one for an impulsive control strategy. In particular, we establish the existence condition under which the system has a locally asymptotically stable prey-eradication periodic solution. Violating such a condition, the system turns out to be permanent. Employing bifurcation theory, some conditions, under which the existence and stability of a positive periodic solution of the system occur but its prey-eradication periodic solution becomes unstable, are provided. Furthermore, numerical simulations for the proposed model are given to confirm the obtained theoretical results.
\end{abstract}

Keywords: Predator-prey system; Anti-predator behavior; Impulsive control strategy; Generalized Holling type IV functional response

\section{Introduction}

Natural phenomena such as porous medium problems and periodically oscillatory waves that we usually observe in science, engineering or some other fields can be explained via mathematical models which are often formulated by means of a differential equation or a system of differential equations [1-3]. However, a relationship between predators and preys in an ecological system is considered as one of the most interesting natural phenomena because it is a type of the most fundamental biological systems in nature. Due to its universal existence and importance, the dynamical behaviors of predator-prey systems have been studied by a great number of scholars [4-7]. Interactions between predator and prey populations are extensively exploited for arranging economically damaging prey (pest) species in an eco-friendly community. In 1925-1926, Lotka and Volterra described the relationship between predators and their preys in an ecological system by establishing a system of two autonomous ordinary differential equations, namely the Lotka-Volterra model. The Lotka-Volterra system is the simplest model of predator-prey interactions

(c) The Author(s) 2021. This article is licensed under a Creative Commons Attribution 4.0 International License, which permits use, sharing, adaptation, distribution and reproduction in any medium or format, as long as you give appropriate credit to the original author(s) and the source, provide a link to the Creative Commons licence, and indicate if changes were made. The images or other third party material in this article are included in the article's Creative Commons licence, unless indicated otherwise in a credit line to the material. If material is not included in the article's Creative Commons licence and your intended use is not permitted by statutory regulation or exceeds the permitted use, you will need to obtain permission directly from the copyright holder. To view a copy of this licence, visit http://creativecommons.org/licenses/by/4.0/ 
based on linear per capita growth rates $[8,9]$. Some recent developments of complex predator-prey systems, which have been investigated in depth by many researchers to obtain their dynamics, are as follows. For example, Tiwari et al. [10] proposed and analyzed a mathematical model for a predator-prey system with multiple Allee effect acting on the growth rate of the prey population. Haldar et al. [11] formulated a delay-induced ecoepidemic model using a reconstructed Leslie-Gower-type growth rate and then analyzed the dynamics of the system with infection in the prey population. A predator-prey model with the additive Allee effect and the fear effect in the prey was presented and investigated by Lai et al. [12]. The bifurcation analysis of the model was explored by considering on the impact of these two effects. To further grasp other crucial predator-prey mathematical models in detail, one can refer to [13-19].

It has been found that the prey adopts several mechanisms for overcoming the predation pressure in their interactions. Two such techniques, which are used in the predator-prey models for an ecological system but not well scrutinized in the literature, are group defense and anti-predator behavior in prey [20]. Firstly, group defense [21-24] is a term used to describe the phenomenon whereby predation is decreased or even prevented altogether because of the enlarged ability of the prey to maintain its own survival via better defending or disguising itself when a sufficiently large number of prey appear. The explorations for predator-prey models with group defense were considered by many researchers such as in [24-27]. Particularly, Freedman and Wolkowicz introduced a general model of predatorprey interactions in which the prey behaves a group defense and there is no mutual interference among predators. Their model consisting of autonomous ordinary differential equations of generalized Gauss-type can be expressed as follows:

$$
\begin{aligned}
& x^{\prime}(t)=x g(x, k)-y p(x), \\
& y^{\prime}(t)=y(-d+q(x)), \\
& x(0) \geq 0, \quad y(0) \geq 0,
\end{aligned}
$$

where $x(t)$ and $y(t)$ represent the density of prey and predator populations, respectively. The functions $g, p$ and $q$ are assumed to be continuously differentiable and the parameters $d$ and $k$ are positive constants. More details as regards system (1) can be found in [21].

Secondly, preys can sometimes jeopardize their predators. This leads to role reversals in predators and prey, namely anti-predator behaviors. Several authors studied the complicated dynamics of predator-prey systems with anti-predator behaviors such as in [20, 2833]. In particular, an anti-predator behavior of prey [20] is a counterattacking approach in which adult preys attack the juvenile predators to decrease their future predation circumstances. Biological experiments demonstrate that anti-predator behaviors of prey populations are characterized as (i) morphological changes or behavior changes [34, 35], or (ii) attacking their predators $[29,36]$. Normally, juvenile preys escaping from predation and becoming adult can counterattack and kill juvenile predators, but do not consume them so that a number of preys probably killed in the future are reduced [37]. Therefore, this cyclic dominance is very significant for predator-prey interactions because there are adverse effects on the biological control strategy. Here are some recent examples of studies of predator-prey models with anti-predator behaviors. In 2017, Sun et al. [38] introduced a predator-prey model equipped with anti-predator behaviors occurring only 
when the population size of the prey is greater than a threshold. They concluded that a large anti-predator rate induces the prey population to persist but whether we have coexistence between prey and predator populations depends upon the threshold activating anti-predator behavior. In 2019, Prasad et al. [20] analyzed the predator-prey model with an anti-predator behavior in prey to overcome the loss in predator populations via catering the predators with some additional food. However, we are quite interested in studying the effect of anti-predator behavior on the predator-prey system proposed by Tang and Xiao [30]. Their mathematical model is expressed as

$$
\begin{aligned}
& x^{\prime}(t)=r x\left(1-\frac{x}{k}\right)-y \Psi(x), \\
& y^{\prime}(t)=\mu y \Psi(x)-d y-\eta x y,
\end{aligned}
$$

in which the functional response $\Psi(x)$ is specifically chosen to be the simplified MonodHaldane functional response $\Psi(x)=\frac{\beta x}{a+x^{2}}$, where $\beta$ is the capture rate of the predator and $a$ is the reciprocal of group defense in prey. The remaining parameters in system (2) are described as follows: $r$ is the intrinsic growth rate of the prey, $k$ is the prey carrying capacity, $\mu$ is the conversion fraction of prey into predator and $d$ is the natural death rate of the predator population. The parameter $\eta$ is the rate of anti-predator behavior of prey to the predator population in such a way that this behavior reduces the growth of the predator population because the prey population is not mainly fed on the predator population. All of the parameter values in system (2) are assumed to be positive. Actually, there are many types of functional response $\Psi(x)$ indicating the change in the density of prey attacked per unit of time per predator when the prey density changes. Some interesting functional responses, widely used in predator-prey models, are Holling type, sigmoid type and Beddington-DeAngelis functional responses. More details as regards the functional responses can be found in [23, 39-41]. However, the generalized Holling type IV functional response or the Monod-Haldane function [5, 42-45]

$$
\Psi(x)=\frac{\beta x}{a+b x+x^{2}},
$$

where $\beta, a, b$ are positive constants, will be selected to use in our work due to its nonmonotonicity.

Next, we adopt the predator-prey model with anti-predator behavior (2) via replacing $\Psi(x)$ with the generalized Holling type IV functional response in Eq. (3). As mentioned, we obtain the following autonomous system:

$$
\begin{aligned}
& x^{\prime}(t)=r x\left(1-\frac{x}{k}\right)-\frac{\beta x y}{a+b x+x^{2}}, \\
& y^{\prime}(t)=\frac{\mu \beta x y}{a+b x+x^{2}}-d y-\eta x y .
\end{aligned}
$$

System (4) can be thought of as adding the anti-predator term, $-\eta x y$, to the predator-prey model with prey group defense in Eq. (1) from which the functions $g, p$ and $q$ are identified as

$$
g(x, k)=r\left(1-\frac{x}{k}\right), \quad p(x)=\frac{\beta x}{a+b x+x^{2}}, \quad \text { and } \quad q(x)=\mu p(x) .
$$


The functions $g, p$ and $q$ in (5) satisfy the conditions (2)-(4) given in [21], respectively, with $M=\sqrt{a}$.

Defining $Q(x)=q(x)-\eta x$, we obtain

$$
Q^{\prime}(x)=\frac{-1}{\left(a+b x+x^{2}\right)^{2}}\left[\eta x^{4}+2 b \eta x^{3}+\left(\eta b^{2}+2 a \eta+\beta \mu\right) x^{2}+2 a b \eta x+a(a \eta-\beta \mu)\right] .
$$

Using Descartes' rule of signs, if $a \eta<\beta \mu$, then the equation $Q^{\prime}(x)=0$ has exactly one positive real root denoted by $x=M^{*}$. It is not difficult to see that $Q(x), x \in[0, \infty)$ has a global maximum at $M^{*}$. Now, we must assume for system (4) that $Q\left(M^{*}\right)>d$ because of otherwise the predator cannot survive on the prey at any density. The predator-prey system (4) will be a part of our impulsive problem proposed later on.

The remaining parts of this paper are organized as follows. In Sect. 2, an impulsive differential system based on the predator-prey model (4) is formulated. The meaning of the state variables and the parameters of the system is given there. Section 3 provides the theoretical outcomes of the impulsive model including the basic results, the extinction and permanence and the bifurcation and existence of positive periodic solution obtained using the Floquet theory of an impulsive system and bifurcation theory. Numerical results showing that the system can exhibit several interesting phenomena are given in Sect. 4 . We discuss and conclude our results in the last section.

\section{Impulsive model formulation}

Over the last few decades, impulsive differential equations have been found in almost every region of applied science and have been studied in many fields such as impulsive birth [46], impulsive vaccination [47], chemotherapeutic treatment of disease [48] and population ecology $[49,50]$. In general, they are exploited to describe the phenomena depending upon steep or instantaneous changes which make the differential system more intractable. A large number of researchers have suggested to apply an impulsive control strategy [5054] to a modified predator-prey model for obtaining some desired results such as a reduction in pest populations in a farm. This approach is known as integrated pest management (IPM), which is a suitable method for manipulating pests (or preys) with a combination of biological, cultural, physical and chemical tools so that one can minimize economic, health and environmental risks $[51,55]$. Periodically releasing natural enemies or spraying pesticides at a different fixed time is regarded as a certain example of the IPM.

The present section is devoted to formulating an impulsive mathematical model for predator-prey model (4) in which the generalized Holling type IV functional response and the anti-predator behavior are inserted. Our impulsive differential system with a fixed moment can be expressed as

$$
\begin{aligned}
& x^{\prime}(t)=r x\left(1-\frac{x}{k}\right)-\frac{\beta x y}{a+b x+x^{2}}, \quad t \neq n T, \\
& y^{\prime}(t)=\frac{\mu \beta x y}{a+b x+x^{2}}-d y-\eta x y, \quad t \neq n T, \\
& \Delta x(t)=-p_{1} x(t), \quad t=n T, \\
& \Delta y(t)=-p_{2} y(t)+p_{3}, \quad t=n T,
\end{aligned}
$$


Table 1 Descriptive list of the variables and parameters appearing in system (7)

\begin{tabular}{lll}
\hline Parameter & Dimension & Description \\
\hline$t$ & time & Time \\
$x$ & biomass & Prey density \\
$y$ & biomass & Predator density \\
$r$ & time $^{-1}$ & Prey intrinsic growth rate \\
$k$ & biomass $^{-1}$ & Prey carrying capacity \\
$\beta$ & biomass $\cdot$ time $^{-1}$ & Capture rate of the predator \\
$a$ & biomass $^{2}$ & Reciprocal of group defense in prey \\
$b$ & biomass $_{\mu}$ & Reciprocal of group defense in prey associated with the prey $x$ \\
$d$ & - & Conversion fraction of the prey into the predator \\
$\eta$ & time $^{-1}$ & Natural death rate of the predator \\
$p_{1}$ & biomass $^{-1} \cdot$ time $^{-1}$ & Rate of anti-predator behavior of the prey \\
$p_{2}$ & - & Proportional constant of the prey which dies due to the pesticide \\
$p_{3}$ & - & Proportional constant of the predator which dies due to the pesticide \\
\hline
\end{tabular}

where $\Delta x(t)=x\left(t^{+}\right)-x(t), \Delta y(t)=y\left(t^{+}\right)-y(t)$ and $T$ is the impulsive period for releasing predators in order to remove target pests, prevent harmless pests from extinction and drive target pests to extinction, or control target pests at acceptably low level to avoid an increase of pest population due to an economic loss. The notations $x\left(t^{+}\right)$and $y\left(t^{+}\right)$are the right limits representing the values of $x$ and $y$ immediately after a pulse at time $t$. The number $n \in \mathbb{N}=\{1,2,3, \ldots\}$ and $p_{1}, p_{2}, p_{3}$ are nonnegative constants with $0 \leq p_{1}, p_{2}<1$, $p_{3} \geq 0$. Here, $p_{1}$ and $p_{2}$ represent the proportional constants of the prey and predator, respectively which are eradicated by using chemical poisons in agriculture or by harvesting. The constant $p_{3}$ is the density of predators released each time. Consequently, the last two equations of Eq. (7) can be considered as the IPM of the system. The values of the parameters $r, k, \beta, \mu, a, b, d, \eta$ are assumed to be positive. The descriptive list of the variables and parameters presented in system (7) is shown in Table 1. To the best of the authors' knowledge, the impulsive system (7) has never been proposed and investigated before so the dynamics of the system remains unclear, and falls within the scope of our exploration.

\section{Analysis of the model}

With a view on the use of the generalized Holling type IV functional response, the antipredator behavior and the impulsive IPM appearing in the impulsive predator-prey system (7), we are interested in analyzing some important properties of the system in this section.

\subsection{Fundamental results}

In the following section, we will show that a solution of system (7) is nonnegative and bounded above for sufficient large $t$. In addition, the system has a prey-eradication periodic solution.

Let $\mathbb{R}_{+}=[0, \infty), \mathbb{R}_{+}^{2}=\left\{z=(x, y) \in \mathbb{R}^{2}: x, y \in \mathbb{R}_{+}\right\}$. Let $F=\left(F_{1}, F_{2}\right)$ denote the map defined by the right hand of system (7). Then a function $V: \mathbb{R}_{+} \times \mathbb{R}_{+}^{2} \rightarrow \mathbb{R}_{+}$is said to belong to class $V_{0}$ if [56]:

(i) $V$ is continuous in $(t, z) \in(n T,(n+1) T] \times \mathbb{R}_{+}^{2}$ and for each $z \in \mathbb{R}_{+}^{2}, n \in \mathbb{N}$,

$$
\lim _{(t, v) \rightarrow\left(n T^{+}, z\right)} V(t, v)=V\left(n T^{+}, z\right)
$$

exists. 
(ii) $V$ is locally Lipschitzian in $z$.

Definition 3.1 Let $V \in V_{0}$. Then, for $(t, z) \in(n T,(n+1) T] \times \mathbb{R}_{+}^{2}$, the upper right derivative of $V(t, z)$ with respect to the impulsive differential system (7) is defined as

$$
D^{+} V(t, z)=\lim _{h \rightarrow 0^{+}} \sup \frac{1}{h}[V(t+h, z+h F(t, z))-V(t, z)] .
$$

The solution $z(t)=(x(t), y(t))$ of system (7) is a piecewise continuous function with the properties that $z: \mathbb{R}^{+} \rightarrow \mathbb{R}_{+}^{2}$ is continuous on $(n T,(n+1) T], n \in \mathbb{N}$, and $z\left(n T^{+}\right)=$ $\lim _{t \rightarrow n T^{+}} z(t)$ exists. The smoothness properties of $F$ ensure the global existence and uniqueness of solutions of system (7). More details can be found in [57]. Note from system (7) that $\frac{d x(t)}{d t}=0$ and $\frac{d y(t)}{d t}=0$ whenever $x(t)=0$ and $y(t)=0$ for $t \neq n T$ and $x\left(n T^{+}\right)=\left(1-p_{1}\right) x(n T)$ and $y\left(n T^{+}\right)=\left(1-p_{2}\right) y(n T)+p_{3}$ where $0 \leq p_{1}, p_{2}<1, p_{3} \geq 0$. Then we have the following lemma.

Lemma 3.1 Suppose $z(t)=(x(t), y(t))$ is a solution of system (7) with $z\left(0^{+}\right) \geq 0$. Then $z(t) \geq$ 0 for all $t \geq 0$. Moreover, $z(t)>0$ for all $t \geq 0$ if $z\left(0^{+}\right)>0$.

Proof The proof by contradiction is here performed. Suppose that there exists $\hat{t} \in(0, T]$ such that $x(t) \geq 0, y(t) \geq 0, x(\hat{t})=0, x^{\prime}(\hat{t})<0$ and $y(\hat{t}) \geq 0$ for all $t \in(0, \hat{t})$. From the first equation of system (7), we have $x^{\prime}(\hat{t})=0$, which is a contradiction. Furthermore, we also obtain

$$
x(t)=x\left(0^{+}\right) \exp \left(\int_{0}^{t}\left[r\left(1-\frac{x(\tau)}{k}\right)-\frac{\beta y(\tau)}{a+b x(\tau)+x(\tau)^{2}}\right] d \tau\right),
$$

where $t \in(0, n T]$. Hence, $x(t) \geq 0$ if $x\left(0^{+}\right) \geq 0$ and $x(t)>0$ if $x\left(0^{+}\right)>0$ for $t \in(0, T]$. The proof for $y(t)$ can be conducted in a like manner.

Next, we show that all solutions of system (7) are uniformly ultimately bounded.

Lemma 3.2 There exists a constant $M>0$ such that $x(t) \leq M$ and $y(t) \leq M$ for each solution $z(t)=(x(t), y(t))$ of system (7) for sufficient large $t$.

Proof Let $V(t, z(t))=\mu x(t)+y(t)$. It is clear that $V \in V_{0}$. Choosing a positive constant $c$ such that

$$
0<c \leq d
$$

and using the upper right derivative of $V(t, z(t))$ along a solution of system (7), we obtain for $t \neq n T$

$$
\begin{aligned}
\left.D^{+} V\right|_{(7)}+c V= & \mu \frac{d x}{d t}+\frac{d y}{d t}+c \mu x+c y \\
= & r \mu x\left(1-\frac{x}{k}\right)-\frac{\mu \beta x y}{a+b x_{1}+x_{1}^{2}}+\frac{\mu \beta x y}{a+b x_{1}+x_{1}^{2}} \\
& -d y-\eta x y+c \mu x+c y
\end{aligned}
$$




$$
\begin{aligned}
& =(r+c) x-\frac{r x^{2}}{k}+(c-d) y-\eta x y \\
& \leq M_{0}
\end{aligned}
$$

where $M_{0} \equiv \frac{k(r+c)^{2}}{4 r}$. Hence, $D^{+} V \leq-c V+M_{0}$ for $t \neq n T$.

For $t=n T$,

$$
\begin{aligned}
V\left(n T^{+}\right) & =\mu x\left(n T^{+}\right)+y\left(n T^{+}\right) \\
& =\left(1-p_{1}\right) \mu x(n T)+\left(1-p_{2}\right) y(n T)+p_{3} \\
& \leq \mu x(n T)+y(n T)+p_{3} \\
& =V(n T)+p_{3} .
\end{aligned}
$$

Hence, for $t \in(n T,(n+1) T]$ and $t_{k}=k T, k=1,2, \ldots, n$, Lemma 2.2 of [58] implies that

$$
\begin{aligned}
V(t) & \leq V(0) e^{-c t}+\int_{0}^{t} M_{0} e^{-c(t-s)} d s+p_{3} \sum_{0<t_{k}<t} e^{\int_{t_{k}}^{t}-c d \tau} \\
& =V(0) e^{-c t}+\frac{M_{0}}{c}\left(1-e^{-c t}\right)+p_{3}\left(\frac{e^{-c(t-T)}-e^{-c(t-(n+1) T)}}{1-e^{c T}}\right) \\
& \rightarrow \frac{M_{0}}{c} \equiv M \quad \text { as } t \rightarrow \infty .
\end{aligned}
$$

Therefore, $V(t, z)$ is ultimately bounded by $M>0$. In consequence, $x(t) \leq M$ and $y(t) \leq$ $M$ when $t$ is sufficiently large. In other words, the positive solution $z(t)$ of system (7) is uniformly ultimately bounded. This completes the proof.

The following comparison theorem on impulsive differential systems [57] will be used for system (7).

Lemma 3.3 Suppose $V \in V_{0}$. Assume that

$$
\begin{aligned}
& D^{+} V(t, z) \leq g(t, V(t, z)), \quad t \neq n T, \\
& V\left(t, z\left(t^{+}\right)\right) \leq h_{n}(V(t, z)), \quad t=n T,
\end{aligned}
$$

where $g: \mathbb{R}_{+} \times \mathbb{R}_{+} \rightarrow \mathbb{R}$ is continuous in $(n T,(n+1) T] \times \mathbb{R}_{+}$, and for $u \in \mathbb{R}_{+}, n \in \mathbb{N}$, $\lim _{(t, v) \rightarrow\left(n T^{+}, u\right)} g(t, v)=g\left(n T^{+}, u\right)$ exists and $h_{n}: \mathbb{R}_{+} \rightarrow \mathbb{R}_{+}$is nondecreasing.

Let $R(t)$ be the maximal solution of the scalar impulsive differential equation

$$
\begin{aligned}
& u^{\prime}(t)=g(t, u(t)), \quad t \neq n T, \\
& u\left(t^{+}\right)=h_{n}(u(t)), \quad t=n T, \\
& u\left(0^{+}\right)=u_{0},
\end{aligned}
$$

existing on $[0, \infty)$. Then $V\left(0^{+}, z\left(0^{+}\right)\right) \leq u_{0}$ implies that $V(t, z(t)) \leq R(t)$ for all $t \geq 0$, where $z(t)$ is any solution of system (7).

Let $r(t)$ be the minimal solution of $(16)$ existing on $[0, \infty)$, and assume that the inequalities in (15) are reversed and $h_{n}$ is nonincreasing. Then $V(t, z(t)) \geq r(t)$ for all $t \geq 0$. 
Note that if we have some smoothness conditions on $g$ to ensure the existence and uniqueness of solutions for (16), then $R(t)$ and $r(t)$ are exactly the unique solution of (16). Consider the following system:

$$
\begin{aligned}
& y^{\prime}(t)=-d y(t), \quad t \neq n T, \\
& \Delta y(t)=-p_{2} y(t)+p_{3}, \quad t=n T, \\
& y\left(0^{+}\right)=y_{0} \geq 0,
\end{aligned}
$$

which is a subsystem of system (7). Some important properties of subsystem (17) are given as follows. It is not difficult to see that

$$
\tilde{y}(t)=\frac{p_{3} \exp (-d(t-n T))}{1-\left(1-p_{2}\right) \exp (-d T)}
$$

is a positive solution of system (17) for $t \in(n T,(n+1) T], n \in \mathbb{N}$ with

$$
\tilde{y}\left(0^{+}\right)=\frac{p_{3}}{1-\left(1-p_{2}\right) \exp (-d T)} .
$$

Since

$$
y(t)=\left(y_{0}-\frac{p_{3}}{1-\left(1-p_{2}\right) \exp (-d T)}\right) \exp (-d t)+\tilde{y}(t)
$$

is the solution of system (17) for $t \in(n T,(n+1) T]$ where $n \in \mathbb{N}$, we have the following lemma.

Lemma 3.4 System (17) has a positive periodic solution $\tilde{y}(t)$ with a period $T$. For every solution $y(t)$ of (17) with $y_{0} \geq 0$, we have $|y(t)-\tilde{y}(t)| \rightarrow 0$ as $t \rightarrow \infty$. Therefore, system (7) has a prey-eradication periodic solution

$$
(0, \tilde{y}(t))=\left(0, \frac{p_{3} \exp (-d(t-n T))}{1-\left(1-p_{2}\right) \exp (-d T)}\right)
$$

for $t \in(n T,(n+1) T]$, and $\tilde{y}\left(n T^{+}\right)=\tilde{y}\left(0^{+}\right)=\frac{p_{3}}{1-\left(1-p_{2}\right) \exp (-d T)}, n \in \mathbb{N}$.

\subsection{Extinction and permanence}

Before studying the permanence of system (7), we will give the condition ensuring the locally asymptotical stability of the prey-eradication periodic solution $(0, \tilde{y}(t))$. Next, the condition for the permanence of the system will be given.

Theorem 3.1 Let $(x(t), y(t))$ be any solution of system $(7)$. Then $(0, \tilde{y}(t))$ is locally asymptotically stable provided that

$$
r T-\frac{\beta p_{3}(1-\exp (-d T))}{a d\left(1-\left(1-p_{2}\right) \exp (-d T)\right)}<\ln \left(\frac{1}{1-p_{1}}\right) .
$$

Proof The local stability of the periodic solution $(0, \tilde{y}(t))$ in Eq. (21) may be determined by scrutinizing the behavior of small amplitude perturbations of the solution. Defining

$$
x(t)=u(t), y(t)=v(t)+\tilde{y}(t),
$$


then we may express

$$
\left(\begin{array}{l}
u(t) \\
v(t)
\end{array}\right)=\Phi(t)\left(\begin{array}{l}
u(0) \\
v(0)
\end{array}\right), \quad 0 \leq t<T,
$$

where $\Phi(t)$ is the fundamental solution matrix satisfying

$$
\Phi^{\prime}(t)=\left(\begin{array}{cc}
r-\frac{\beta}{a} \tilde{y}(t) & 0 \\
\left(\frac{\mu \beta}{a}-\eta\right) \tilde{y}(t) & -d
\end{array}\right) \Phi(t)
$$

and $\Phi(0)=I$, the $2 \times 2$ identity matrix. Hence,

$$
\Phi(t)=\left(\begin{array}{cc}
\exp \left(\int_{0}^{t}\left(r-\frac{\beta}{a} \tilde{y}(s)\right) d s\right) & 0 \\
* & \exp (-d t)
\end{array}\right) .
$$

The term $(*)$ will not be used in further calculation; then there is no need to calculate the exact expression for $(*)$ in the above equation.

The linearization of the last two equations of system (7) becomes

$$
\left(\begin{array}{l}
u\left(n T^{+}\right) \\
v\left(n T^{+}\right)
\end{array}\right)=\left(\begin{array}{cc}
1-p_{1} & 0 \\
0 & 1-p_{2}
\end{array}\right)\left(\begin{array}{l}
u(n T) \\
v(n T)
\end{array}\right) .
$$

According to the Floquet theory [58] of an impulsive differential equation, if both eigenvalues of the matrix

$$
\mathscr{M}=\left(\begin{array}{cc}
1-p_{1} & 0 \\
0 & 1-p_{2}
\end{array}\right) \Phi(T)
$$

have absolute values less than one, then the periodic solution $(0, \tilde{y}(t))$ is locally asymptotically stable.

Let $\lambda_{1}$ and $\lambda_{2}$ denote the eigenvalues of $\mathscr{M}$. We have

$$
\lambda_{1}=\left(1-p_{2}\right) \exp (-d T)
$$

and

$$
\begin{aligned}
\lambda_{2} & =\left(1-p_{1}\right) \exp \left(\int_{0}^{T}\left(r-\frac{\beta}{a} \tilde{y}(t)\right) d t\right) \\
& =\left(1-p_{1}\right) \exp \left(r T-\frac{p_{3} \beta(1-\exp (-d T))}{a d\left(1-\left(1-p_{2}\right) \exp (-d T)\right)}\right)
\end{aligned}
$$

It is obvious that $\left|\lambda_{1}\right|<1$. However, $\left|\lambda_{2}\right|<1$ if and only if

$$
r T-\frac{\beta p_{3}(1-\exp (-d T))}{a d\left(1-\left(1-p_{2}\right) \exp (-d T)\right)}<\ln \left(\frac{1}{1-p_{1}}\right) .
$$

Hence, the solution $(0, \tilde{y}(t))$ of system $(7)$ is locally asymptotically stable when the condition (31) holds. This completes the proof. 
Definition 3.2 System (7) is said to be permanent if there exist positive constants $m, M$ and $T_{0}$ such that each positive solution $z(t)=(x(t), y(t))$ of system (7) with the initial values $x\left(0^{+}\right)>0$ and $y\left(0^{+}\right)>0$ satisfies $m \leq x(t) \leq M$ and $m \leq y(t) \leq M$ for all $t \geq T_{0}$. Note that $m, M$ are independent of the initial values but $T_{0}$ may depend on the initial values.

Theorem 3.2 System (7) is permanent if the condition

$$
r T-\frac{\beta p_{3}(1-\exp (-d T))}{a d\left(1-\left(1-p_{2}\right) \exp (-d T)\right)}>\ln \left(\frac{1}{1-p_{1}}\right)
$$

holds.

Proof Suppose that $z(t)=(x(t), y(t))$ is a solution of system (7) with $x\left(0^{+}\right)>0, y\left(0^{+}\right)>0$. Then Lemma 3.2 guarantees that $z(t)$ is bounded above when $t$ is large enough. Consequently, there is a constant $M>\frac{r a}{\beta}>0$ such that $x(t) \leq M$ and $y(t) \leq M$ when $t$ is sufficiently large.

Since $\frac{\mu \beta x y}{a+b x_{1}+x_{1}^{2}} \geq 0$, system (7) implies that

$$
\begin{aligned}
& y^{\prime}(t) \geq-D_{0} y(t), \quad t \neq n T, \\
& y\left(t^{+}\right)=\left(1-p_{2}\right) y(t)+p_{3}, \quad t=n T, \\
& y\left(0^{+}\right)=y_{0} \geq 0,
\end{aligned}
$$

where $D_{0} \equiv d+\eta M>0$. Consider the comparison system

$$
\begin{aligned}
& s^{\prime}(t)=-D_{0} s(t), \quad t \neq n T, \\
& s\left(t^{+}\right)=\left(1-p_{2}\right) s(t)+p_{3}, \quad t=n T, \\
& s\left(0^{+}\right)=y_{0} .
\end{aligned}
$$

We see that, for $t \in(n T,(n+1) T], n \in \mathbb{N}$,

$$
\tilde{s}(t)=\frac{p_{3} \exp \left(-D_{0}(t-n T)\right)}{1-\left(1-p_{2}\right) \exp \left(-D_{0} T\right)}
$$

is a positive solution of the comparison system (34) with

$$
\tilde{s}\left(0^{+}\right)=\frac{p_{3}}{1-\left(1-p_{2}\right) \exp \left(-D_{0} T\right)} .
$$

Therefore, the solution of the comparison system (34) is

$$
s(t)=\left(y_{0}-\frac{p_{3}}{1-\left(1-p_{2}\right) \exp \left(-D_{0} T\right)}\right) \exp \left(-D_{0} t\right)+\tilde{s}(t),
$$

for $t \in(n T,(n+1) T], n \in \mathbb{N}$ and $s(t) \rightarrow \tilde{s}(t)$ as $t \rightarrow \infty$. Then we have

$$
s(t)>\tilde{s}(t)-\varepsilon_{2},
$$


for some $\varepsilon_{2}>0$ and for all sufficiently large $t$. According to [57], we have $y(t) \geq s(t)$ by Lemma 3.3 and hence, when $t$ is large enough,

$$
y(t) \geq \frac{p_{3} \exp \left(-D_{0} T\right)}{1-\left(1-p_{2}\right) \exp \left(-D_{0} T\right)}-\varepsilon_{2} \equiv m_{2}>0 .
$$

Then we only need to find a constant $m_{1}>0$ such that $x(t) \geq m_{1}$ for all $t$ large enough. Following the two steps below, we will obtain the desired result.

Step 1. From condition (32), we can equivalently get

$$
\left(1-p_{1}\right) \exp \left[r T-\frac{\beta p_{3}(1-\exp (-d T))}{a d\left(1-\left(1-p_{2}\right) \exp (-d T)\right)}\right]>1 .
$$

Based on condition (40), one can choose $m_{3}>0$ and $\varepsilon_{1}>0$ sufficiently small such that $m_{3}<\min \left\{k, \frac{a d}{\mu \beta}\right\}, D_{1} \equiv d-\frac{\mu \beta m_{3}}{a}>0$ and

$$
\begin{aligned}
\Omega \equiv & \left(1-p_{1}\right) \\
& \times \exp \left[\left(r-\frac{r}{k} m_{3}-\frac{\beta}{a} \varepsilon_{1}\right) T-\frac{\beta}{a}\left(\frac{p_{3}\left(1-\exp \left(-D_{1} T\right)\right)}{D_{1}\left(1-\left(1-p_{2}\right) \exp \left(-D_{1} T\right)\right)}\right)\right]>1 .
\end{aligned}
$$

We will prove that $x(t)<m_{3}$ cannot hold for all $t \geq 0$. Otherwise, from the second and fourth equations of system (7) and the fact that $x^{2}+b x \geq 0$ and $\eta x \geq 0$ due to $x(t) \geq 0$, we consequently have

$$
\begin{aligned}
y^{\prime}(t) & \leq y(t)\left(\frac{\mu \beta x(t)}{a}-d-\eta x(t)\right) \\
& \leq y(t)\left(\frac{\mu \beta x(t)}{a}-d\right) \\
& <y(t)\left(\frac{\mu \beta m_{3}}{a}-d\right) \\
& =-D_{1} y(t), \quad t \neq n T, \\
y\left(t^{+}\right) & =\left(1-p_{2}\right) y(t)+p_{3}, \quad t=n T .
\end{aligned}
$$

From Lemmas 3.3 and 3.4, we obtain $y(t) \leq w(t)$ and $w(t) \rightarrow \tilde{w}(t)$ as $t \rightarrow \infty$, where $w(t)$ is the solution of the following system:

$$
\begin{aligned}
& w^{\prime}(t)=-D_{1} w(t), \quad t \neq n T, \\
& w\left(t^{+}\right)=\left(1-p_{2}\right) w(t)+p_{3}, \quad t=n T, \\
& w\left(0^{+}\right)=y\left(0^{+}\right),
\end{aligned}
$$

and

$$
\tilde{w}(t)=\frac{p_{3} \exp \left(-D_{1}(t-n T)\right)}{1-\left(1-p_{2}\right) \exp \left(-D_{1} T\right)}, \quad t \in(n T,(n+1) T] .
$$

Therefore, there exists a bound $T_{1}>0$ such that

$$
y(t) \leq w(t)<\tilde{w}(t)+\varepsilon_{1}
$$


and

$$
\begin{aligned}
x^{\prime}(t) & =x(t)\left(r\left(1-\frac{x(t)}{k}\right)-\frac{\beta y(t)}{a+b x(t)+x^{2}(t)}\right) \\
& >x(t)\left(r\left(1-\frac{m_{3}}{k}\right)-\frac{\beta}{a}\left(\tilde{w}(t)+\varepsilon_{1}\right)\right), \quad t \neq n T, \\
x\left(t^{+}\right) & =\left(1-p_{1}\right) x(t), \quad t=n T,
\end{aligned}
$$

for $t \geq T_{1}$.

Let $k \in \mathbb{N}$ and $k T \geq T_{1}$. Integrating (46) on $(n T,(n+1) T], n \geq k$, we obtain

$$
\begin{aligned}
x((n+1) T)> & x\left(n T^{+}\right) \exp \left[\int_{n T}^{(n+1) T}\left(r-\frac{r m_{3}}{k}-\frac{\beta}{a}\left(\tilde{w}(t)+\varepsilon_{1}\right)\right) d t\right] \\
= & x(n T)\left(1-p_{1}\right) \exp \left[r T-\frac{r}{k} m_{3} T\right. \\
& \left.-\frac{\beta}{a} \varepsilon_{1} T-\frac{\beta}{a}\left(\frac{p_{3}\left(1-\exp \left(-D_{1} T\right)\right)}{D_{1}\left(1-\left(1-p_{2}\right) \exp \left(-D_{1} T\right)\right)}\right)\right] \\
= & x(n T) \Omega,
\end{aligned}
$$

where $\Omega$ is expressed in Eq. (41). Then $x((n+k) T)>x(n T) \Omega^{k} \rightarrow \infty$ as $k \rightarrow \infty$, which contradicts the boundedness of $x(t)$. Hence, there exists a time $t_{1}>0$ such that $x\left(t_{1}\right) \geq m_{3}$.

Step 2. If $x(t) \geq m_{3}$ for all $t \geq t_{1}$, then our aim is attained. Otherwise, let $t^{*}=\inf _{t>t_{1}}\{t$ : $\left.x(t)<m_{3}\right\}$. Next, we consider two possible cases for $t^{*}$.

Case 1. $t^{*}=n_{1} T, n_{1} \in \mathbb{N}$. Then $x(t) \geq m_{3}$ for $t \in\left[t_{1}, t^{*}\right]$ and

$$
\left(1-p_{1}\right) m_{3} \leq\left(1-p_{1}\right) x\left(t^{*}\right)=x\left(t^{*+}\right)<m_{3} .
$$

Choose $n_{2}, n_{3} \in \mathbb{N}$ such that

$$
\begin{aligned}
& n_{2} T>T_{2} \equiv \frac{1}{-D_{1}} \ln \left(\frac{\varepsilon_{1}}{M+p_{3}}\right), \\
& \left(1-p_{1}\right)^{n_{2}} \exp \left(n_{2} \Omega_{1} T\right) \Omega^{n_{3}}>\left(1-p_{1}\right)^{n_{2}} \exp \left(\left(n_{2}+1\right) \Omega_{1} T\right) \Omega^{n_{3}}>1,
\end{aligned}
$$

where

$$
\Omega_{1} \equiv r-\frac{r m_{3}}{k}-\frac{\beta M}{a}<0 .
$$

Now we let $T^{\prime}=n_{2} T+n_{3} T$ and claim that $t_{2} \in\left(t^{*}, t^{*}+T^{\prime}\right]$ must exist for which $x\left(t_{2}\right)>m_{3}$. Otherwise, considering system (43) with $w\left(t^{*+}\right)=y\left(t^{*+}\right)$, we have

$$
w(t)=\left(w\left(t^{*+}\right)-\frac{p_{3}}{1-\left(1-p_{2}\right) \exp \left(-D_{1} T\right)}\right) \exp \left(-D_{1}\left(t-t^{*}\right)\right)+\tilde{w}(t),
$$

for $t \in(n T,(n+1) T]$, where $n_{1} \leq n \leq n_{1}+n_{2}+n_{3}$.

Using $w(t)-\tilde{w}(t)<\left(y\left(t^{*+}\right)-p_{3}\right) \exp \left(-D_{1}\left(t-t^{*}\right)\right)$ and the first inequality of condition (49), we then have

$$
|w(t)-\tilde{w}(t)|<\left(M+p_{3}\right) \exp \left(-D_{1}\left(t-t^{*}\right)\right)<\varepsilon_{1}
$$


and

$$
y(t) \leq w(t)<\tilde{w}(t)+\varepsilon_{1}
$$

for $t^{*}+n_{2} T \leq t \leq t^{*}+T^{\prime}$. This implies that (46) holds for $t^{*}+n_{2} T \leq t \leq t^{*}+T^{\prime}$. So as in Step 1 we have

$$
x\left(t^{*}+T^{\prime}\right) \geq x\left(t^{*}+n_{2} T\right) \Omega^{n_{3}},
$$

where $\Omega$ is defined in Eq. (41).

From system (7), we have for $t \in\left[t^{*}, t^{*}+n_{2} T\right]$

$$
\begin{aligned}
& x^{\prime}(t) \geq x(t)\left(r-\frac{r m_{3}}{k}-\frac{\beta M}{a}\right)=\Omega_{1} x(t), \quad t \neq n T, \\
& x\left(t^{+}\right)=\left(1-p_{1}\right) x(t), \quad t=n T,
\end{aligned}
$$

where $\Omega_{1}$ is defined in Eq. (50). Integrating (55) on $\left[t^{*}, t^{*}+n_{2} T\right]$, we obtain

$$
x\left(t^{*}+n_{2} T\right) \geq x\left(t^{*+}\right) \exp \left(\int_{t^{*}}^{t^{*}+n_{2} T} \Omega_{1} d t\right)=x\left(t^{*}\right)\left(1-p_{1}\right) \exp \left(\Omega_{1} n_{2} T\right) .
$$

Since $\left(1-p_{1}\right) \geq\left(1-p_{1}\right)^{n_{2}}, n_{2} \in \mathbb{N}$ and $x\left(t^{*}\right) \geq m_{3}$, we have

$$
x\left(t^{*}+n_{2} T\right) \geq m_{3}\left(1-p_{1}\right)^{n_{2}} \exp \left(n_{2} \Omega_{1} T\right) .
$$

From the second condition of (49) along with the inequalities in (54) and (57), we have

$$
x\left(t^{*}+T^{\prime}\right) \geq m_{3}\left(1-p_{1}\right)^{n_{2}} \exp \left(n_{2} \Omega_{1} T\right) \Omega^{n_{3}}>m_{3},
$$

which contradicts the assumption that $x(t) \leq m_{3}$ for all $t \in\left(t^{*}, t^{*}+T^{\prime}\right]$. Thus, there must exist $t_{2} \in\left(t^{*}, t^{*}+T^{\prime}\right]$ so that $x\left(t_{2}\right)>m_{3}$.

Next, we let $\tilde{t}=\inf _{t>t^{*}}\left\{t: x(t)>m_{3}\right\}$. Since $x(t)$ is left continuous and $x\left(t^{+}\right)=(1-p) x(t) \leq$ $x(t)$ when $t=n T$, we can conclude that $x(t) \leq m_{3}$ for $t \in\left(t^{*}, \tilde{t}\right)$ and $x(\tilde{t})=m_{3}$. For $t \in\left(t^{*}, \tilde{t}\right)$, we assume that $t \in\left(t^{*}+(l-1) T, t^{*}+l T\right]$ where $l \in \mathbb{N}$ and $l \leq n_{2}+n_{3}$. From (55) we have

$$
\begin{aligned}
x(t) & \geq x\left(t^{*+}\right)\left(1-p_{1}\right)^{l-1} \exp \left((l-1) \Omega_{1} T\right) \exp \left(\Omega_{1}\left(t-\left(t^{*}+(l-1) T\right)\right)\right) \\
& =x\left(t^{*}\right)\left(1-p_{1}\right)^{l} \exp \left((l-1) \Omega_{1} T\right) \exp \left(\Omega_{1}\left(t-\left(t^{*}+(l-1) T\right)\right)\right) \\
& \geq m_{3}\left(1-p_{1}\right)^{l} \exp \left(l \Omega_{1} T\right) \\
& \geq m_{3}\left(1-p_{1}\right)^{n_{2}+n_{3}} \exp \left(\left(n_{2}+n_{3}\right) \Omega_{1} T\right) \equiv m_{1}^{\prime} .
\end{aligned}
$$

Hence, we can conclude that $x(t) \geq m_{1}^{\prime}$ for $t \in\left(t^{*}, \tilde{t}\right)$. For $t>\tilde{t}$, the same arguments can be continued since $x(\tilde{t}) \geq m_{3}$. In consequence, when $t$ is sufficiently large, we obtain $x(t) \geq$ $m_{1}>0$.

Case 2. $t^{*} \neq n T, n \in \mathbb{N}$. Then $x(t) \geq m_{3}$ for $t \in\left[t_{1}, t^{*}\right)$ and $x\left(t^{*}\right)=m_{3}$. Suppose $t^{*} \in$ $\left(n_{1}^{\prime} T,\left(n_{1}^{\prime}+1\right) T\right), n_{1}^{\prime} \in \mathbb{N}$. The following two possible cases for $t \in\left(t^{*},\left(n_{1}^{\prime}+1\right) T\right)$ are considered. 
Case 2.1. $x(t) \leq m_{3}$ for all $t \in\left(t^{*},\left(n_{1}^{\prime}+1\right) T\right)$. We claim that $t_{2}^{\prime} \in\left[\left(n_{1}^{\prime}+1\right) T,\left(n_{1}^{\prime}+1\right) T+T^{\prime}\right]$ must exist for which $x\left(t_{2}^{\prime}\right)>m_{3}$. Otherwise, considering system (43) with $w\left(\left(n_{1}^{\prime}+1\right) T^{+}\right)=$ $y\left(\left(n^{\prime}+1\right) T^{+}\right)$, then we have

$$
\begin{aligned}
w(t)= & \left(w\left(\left(n_{1}^{\prime}+1\right) T^{+}\right)-\frac{p_{3}}{1-\left(1-p_{2}\right) \exp \left(-D_{1} T\right)}\right) \\
& \times \exp \left(-D_{1}\left(t-\left(n_{1}^{\prime}+1\right) T\right)\right)+\tilde{w}(t),
\end{aligned}
$$

for $t \in(n T,(n+1) T]$ and $n_{1}^{\prime}+1 \leq n \leq n_{1}^{\prime}+1+n_{2}+n_{3}$.

Similarly to Case 1 of Step 2, we have

$$
x\left(\left(n_{1}^{\prime}+1+n_{2}+n_{3}\right) T\right) \geq x\left(\left(n_{1}^{\prime}+1+n_{2}\right) T\right) \Omega^{n_{3}} .
$$

Since $x(t) \leq m_{3}$ for $t \in\left(t^{*},\left(n_{1}^{\prime}+1\right) T\right)$, (55) is true on $\left[t^{*},\left(n_{1}^{\prime}+1+n_{2}\right) T\right]$. Thus, we obtain

$$
x\left(\left(n_{1}^{\prime}+1+n_{2}\right) T\right) \geq m_{3}\left(1-p_{1}\right)^{n_{2}} \exp \left(\left(n_{2}+1\right) \Omega_{1} T\right) .
$$

From the second condition of (49) along with the inequalities in (61) and (62), we thus obtain

$$
x\left(\left(n_{1}^{\prime}+1+n_{2}+n_{3}\right) T\right) \geq m_{3}\left(1-p_{1}\right)^{n_{2}} \exp \left(\left(n_{2}+1\right) \Omega_{1} T\right) \Omega^{n_{3}}>m_{3} .
$$

The contradiction now emerges.

Next, we let $\bar{t}=\inf _{t>t^{*}}\left\{t: x(t)>m_{3}\right\}$. For $t \in\left(t^{*}, \bar{t}\right)$, we have $x(t) \leq m_{3}$ and $x(\bar{t})=m_{3}$. Now we suppose for $t \in\left(t^{*}, \bar{t}\right)$ that $t \in\left(n_{1}^{\prime} T+\left(l^{\prime}-1\right) T, n_{1}^{\prime} T+l^{\prime} T\right)$ where $l^{\prime} \in \mathbb{N}$ and $l^{\prime} \leq n_{2}+n_{3}+1$. From (55), we hence have

$$
\begin{aligned}
x(t) & \geq m_{3}\left(1-p_{1}\right)^{l^{\prime}-1} \exp \left(l^{\prime} \Omega_{1} T\right) \\
& \geq m_{3}\left(1-p_{1}\right)^{n_{2}+n_{3}} \exp \left(\left(n_{2}+n_{3}+1\right) \Omega_{1} T\right) \equiv m_{1},
\end{aligned}
$$

where $m_{1}<m_{1}^{\prime}$. So, $x(t) \geq m_{1}$ for $t \in\left(t^{*}, \bar{t}\right)$. For $t>\bar{t}$, the same arguments can be applied since $x(\bar{t}) \geq m_{3}$. We consequently have $x(t) \geq m_{1}>0$ when $t$ is large enough.

Case 2.2. There exists a time $t \in\left(t^{*},\left(n_{1}^{\prime}+1\right) T\right)$ such that $x(t)>m_{3}$. Let $\check{t}=\inf _{t>t^{*}}\{t: x(t)>$ $\left.m_{3}\right\}$. Therefore, $x(t) \leq m_{3}$ for $t \in\left(t^{*}, \check{t}\right)$ and $x(\check{t})=m_{3}$. We see that (55) holds for $t \in\left(t^{*}, \check{t}\right)$. Integrating (55) on $\left(t^{*}, \check{t}\right)$, we then have

$$
x(t) \geq x\left(t^{*}\right) \exp \left(\Omega_{1}\left(t-t^{*}\right)\right) \geq m_{3} \exp \left(\Omega_{1} T\right)>m_{1} .
$$

The above arguments can be applied again for $t>\check{t}$ using the fact that $x(\check{t}) \geq m_{3}$. Hence, $x(t) \geq m_{1}$ for all $t \geq t_{1}$. In other words, $x(t) \geq m_{1}>0$ when $t$ is sufficiently large.

Finally, we can choose $\hat{m}=\min \left\{m_{1}, m_{2}\right\}$ so that $x(t), y(t) \geq \hat{m}>0$. So, the system is permanent if condition (32) holds. The proof is now complete.

Remark 3.1 Let

$$
g(T)=r T-\frac{\beta p_{3}(1-\exp (-d T))}{a d\left(1-\left(1-p_{2}\right) \exp (-d T)\right)}-\ln \left(\frac{1}{1-p_{1}}\right) .
$$


Since $g(0)=-\ln \left(\frac{1}{1-p_{1}}\right)<0, g(T) \rightarrow \infty$ as $T \rightarrow \infty$ and

$$
g^{\prime \prime}(T)=\frac{\beta d p_{2} p_{3} \exp (-d T)\left(1+\left(1-p_{2}\right) \exp (-d T)\right)}{a\left(1-\left(1-p_{2}\right) \exp (-d T)\right)^{3}}>0,
$$

$g(T)=0$ has a unique positive root, denoted by $T_{\max }$. From Theorems 3.1 and 3.2 we know that $T_{\max }$ is a threshold. If $T<T_{\max }$, then the prey-eradication periodic solution $(0, \tilde{y}(t))$ is asymptotically stable. However, if $T>T_{\max }$, then system (7) is permanent.

\subsection{Bifurcation and stability of positive periodic solution}

In this section, we will investigate the existence of a nontrivial periodic solution to system (7) near the prey-eradication periodic solution $(0, \tilde{y}(t))$ via bifurcation. Such a positive periodic solution is stable when the prey-eradication loses its stability. To do this, we apply Theorem 2 of [59] to system (7) by interchanging the state variables $x(t), y(t)$ and giving a new variable for the period $T$.

Letting $x_{1}(t)=y(t), x_{2}(t)=x(t)$ and $\tau=T$, system (7) consequently becomes

$$
\begin{aligned}
& \frac{d x_{1}(t)}{d t}=\frac{\mu \beta x_{1} x_{2}}{a+b x_{2}+x_{2}^{2}}-d x_{1}-\eta_{1} x_{1} x_{2}, \quad t \neq n \tau, \\
& \frac{d x_{2}(t)}{d t}=r x_{2}\left(1-\frac{x_{2}}{k}\right)-\frac{\beta x_{1} x_{2}}{a+b x_{2}+x_{2}^{2}}, \quad t \neq n \tau, \\
& \Delta x_{1}(t)=-p_{2} x_{1}(t)+p_{3}, \quad t=n \tau, \\
& \Delta x_{2}(t)=-p_{1} x_{2}(t), \quad t=n \tau .
\end{aligned}
$$

All notations used in this section are the same as those in [59]. Then

$$
\begin{aligned}
& F_{1}\left(x_{1}, x_{2}\right) \equiv \frac{\mu \beta x_{1} x_{2}}{a+b x_{2}+x_{2}^{2}}-d x_{1}-\eta x_{1} x_{2}, \\
& F_{2}\left(x_{1}, x_{2}\right) \equiv r x_{2}\left(1-\frac{x_{2}}{k}\right)-\frac{\beta x_{1} x_{2}}{a+b x_{2}+x_{2}^{2}}, \\
& \Theta_{1}\left(x_{1}, x_{2}\right) \equiv\left(1-p_{2}\right) x_{1}+p_{3}, \quad \Theta_{2}\left(x_{1}, x_{2}\right) \equiv\left(1-p_{1}\right) x_{2}, \\
& \varsigma(t)=(\tilde{y}(t), 0)^{T}, \quad X_{0}=\left(\tilde{y}\left(\tau_{0}\right), 0\right)^{T},
\end{aligned}
$$

where $\tilde{y}$ is defined in Eq. (18) with $T=\tau$ and where $\tau_{0}$ is the root of $d_{0}^{\prime} \equiv 1-\left(\frac{\partial \Theta_{2}}{\partial x_{2}} \frac{\partial \Phi_{2}}{\partial x_{2}}\right)_{\left(\tau_{0}, X_{0}\right)}=$ 0 in which $\Phi=\left(\Phi_{1}, \Phi_{2}\right)^{T}$ is the flow associated to system (68). It will be notified in a later step that $\tau_{0}=T_{\max }$ which is the positive root of $g(T)=0$, where $g(T)$ is defined in Eq. (66). As defined above, we have

$$
\begin{aligned}
\frac{\partial F_{1}(\varsigma(u))}{\partial x_{1}} & =\left.\left(\frac{\mu \beta x_{2}}{a+b x_{2}+x_{2}^{2}}-d-\eta x_{2}\right)\right|_{\zeta(u)}=-d, \\
\frac{\partial F_{1}(\zeta(u))}{\partial x_{2}} & =\left.\left(\frac{\mu \beta x_{1}\left(a-x_{2}^{2}\right)}{\left(a+b x_{2}+x_{2}^{2}\right)^{2}}-\eta x_{1}\right)\right|_{\zeta(u)} \\
& =\left(\frac{\mu \beta}{a}-\eta\right) \tilde{y}(u)=\left(\frac{\mu \beta}{a}-\eta\right) \frac{p_{3} \exp (-d(u-n \tau))}{1-\left(1-p_{2}\right) \exp (-d \tau)},
\end{aligned}
$$




$$
\begin{aligned}
\frac{\partial F_{2}(\varsigma(u))}{\partial x_{2}} & =\left.\left(r\left(1-\frac{2 x_{2}}{k}\right)-\left(\frac{\beta x_{1}\left(a-x_{2}^{2}\right)}{\left(a+b x_{2}+x_{2}^{2}\right)^{2}}\right)\right)\right|_{\zeta(u)} \\
& =r-\frac{\beta}{a}\left(\frac{p_{3} \exp (-d(u-n \tau))}{1-\left(1-p_{2}\right) \exp (-d \tau)}\right), \\
\frac{\partial^{2} F_{2}(\varsigma(u))}{\partial x_{1} x_{2}} & =-\left.\left(\frac{\beta\left(a-x_{2}^{2}\right)}{\left(a+b x_{2}+x_{2}^{2}\right)^{2}}\right)\right|_{\zeta(u)}=-\frac{\beta}{a}, \\
\frac{\partial^{2} F_{2}(\varsigma(u))}{\partial x_{2}^{2}} & =\left.\left(-\frac{2 r}{k}+\left(\frac{2 \beta x_{1}\left(a b+3 a x_{2}-x_{2}^{3}\right)}{\left(a+b x_{2}+x_{2}^{2}\right)^{3}}\right)\right)\right|_{\zeta(u)} \\
& =\frac{2 b \beta \tilde{y}(u)}{a^{2}}-\frac{2 r}{k} \\
& =\frac{2 b \beta}{a^{2}}\left(\frac{p_{3} \exp (-d(u-n \tau))}{1-\left(1-p_{2}\right) \exp (-d \tau)}\right)-\frac{2 r}{k} .
\end{aligned}
$$

Next, we obtain the following terms involving the flow $\Phi=\left(\Phi_{1}, \Phi_{2}\right)^{T}$ :

$$
\begin{aligned}
& \frac{\partial \Phi_{1}\left(\tau_{0}, X_{0}\right)}{\partial \tau}=\left.\tilde{y}^{\prime}\left(\tau_{0}\right)\right|_{\left(\tau_{0}, X_{0}\right)}=-\left.\frac{d p_{3} \exp \left(-d\left(\tau_{0}-n \tau\right)\right)}{1-\left(1-p_{2}\right) \exp (-d \tau)}\right|_{\left(\tau_{0}, X_{0}\right)} \\
& =-\frac{d p_{3} \exp \left(-d \tau_{0}\right)}{1-\left(1-p_{2}\right) \exp \left(-d \tau_{0}\right)}<0, \\
& \frac{\partial \Phi_{1}\left(\tau_{0}, X_{0}\right)}{\partial x_{1}}=\exp \left(\int_{0}^{\tau_{0}} \frac{\partial F_{1}(\varsigma(u))}{\partial x_{1}} d u\right)=\exp \left(-d \tau_{0}\right)>0, \\
& \frac{\partial \Phi_{2}\left(\tau_{0}, X_{0}\right)}{\partial x_{2}}=\exp \left(\int_{0}^{\tau_{0}} \frac{\partial F_{2}(\varsigma(u))}{\partial x_{2}} d u\right) \\
& =\exp \left(\int_{0}^{\tau_{0}}\left(r-\frac{\beta \tilde{y}(u)}{a}\right) d u\right) \\
& =\exp \left(r \tau_{0}-\frac{\beta p_{3}\left(1-\exp \left(-d \tau_{0}\right)\right)}{a d\left(1-\left(1-p_{2}\right) \exp \left(-d \tau_{0}\right)\right)}\right) \\
& =\frac{1}{1-p_{1}}>0 \text {, } \\
& \frac{\partial \Phi_{1}\left(\tau_{0}, X_{0}\right)}{\partial x_{2}}=\int_{0}^{\tau_{0}} \exp \left(\int_{v}^{\tau_{0}} \frac{\partial F_{1}(\varsigma(u))}{\partial x_{1}} d u\right)\left(\frac{\partial F_{1}(\varsigma(v))}{\partial x_{2}}\right) \\
& \times \exp \left(\int_{0}^{v} \frac{\partial F_{2}(\varsigma(u))}{\partial x_{2}} d u\right) d v \\
& =\left(\frac{\mu \beta}{a}-\eta\right) \int_{0}^{\tau_{0}} \exp \left(-d\left(\tau_{0}-v\right)\right) \tilde{y}(v) \\
& \times \exp \left(\int_{0}^{v}\left(r-\frac{\beta \tilde{y}(u)}{a}\right) d u\right) d v, \\
& \frac{\partial^{2} \Phi_{2}\left(\tau_{0}, X_{0}\right)}{\partial x_{1} \partial x_{2}}=\int_{0}^{\tau_{0}} \exp \left(\int_{v}^{\tau_{0}} \frac{\partial F_{2}(\varsigma(u))}{\partial x_{2}} d u\right)\left(\frac{\partial^{2} F_{2}(\varsigma(v))}{\partial x_{1} \partial x_{2}}\right) \\
& \times \exp \left(\int_{0}^{v} \frac{\partial F_{2}(\zeta(u))}{\partial x_{2}} d u\right) d v \\
& =-\frac{\beta}{a} \int_{0}^{\tau_{0}} \exp \left(\int_{0}^{\tau_{0}}\left(r-\frac{\beta \tilde{y}(u)}{a}\right) d u\right) d v
\end{aligned}
$$




$$
\begin{aligned}
& =-\frac{\beta \tau_{0}}{a} \exp \left(r \tau_{0}-\frac{\beta p_{3}\left(1-\exp \left(-d \tau_{0}\right)\right)}{a d\left(1-\left(1-p_{2}\right) \exp \left(-d \tau_{0}\right)\right)}\right) \\
& =-\frac{\beta \tau_{0}}{a}\left(\frac{1}{1-p_{1}}\right)<0, \\
& \frac{\partial^{2} \Phi_{2}\left(\tau_{0}, X_{0}\right)}{\partial \tau \partial x_{2}}=\frac{\partial F_{2}\left(\varsigma\left(\tau_{0}\right)\right)}{x_{2}} \exp \left(\int_{0}^{\tau_{0}} \frac{\partial F_{2}(\varsigma(u))}{\partial x_{2}} d u\right) \\
& =\left(r-\frac{\beta \tilde{y}\left(\tau_{0}\right)}{a}\right) \exp \left(\int_{0}^{\tau_{0}}\left(r-\frac{\beta \tilde{y}(u)}{a}\right) d u\right) \\
& =\frac{1}{1-p_{1}}\left(r-\frac{\beta p_{3} \exp \left(-d \tau_{0}\right)}{a\left(1-\left(1-p_{2}\right) \exp \left(-d \tau_{0}\right)\right)}\right) \text {, } \\
& \frac{\partial^{2} \Phi_{2}\left(\tau_{0}, X_{0}\right)}{\partial x_{2}^{2}}=\int_{0}^{\tau_{0}} \exp \left(\int_{v}^{\tau_{0}} \frac{\partial F_{2}(\varsigma(u))}{\partial x_{2}} d u\right)\left(\frac{\partial^{2} F_{2}(\varsigma(v))}{\partial x_{2}^{2}}\right) \\
& \times \exp \left(\int_{0}^{v} \frac{\partial F_{2}(\varsigma(u))}{\partial x_{2}} d u\right) d v \\
& +\int_{0}^{\tau_{0}}\left[\exp \left(\int_{v}^{\tau_{0}} \frac{\partial F_{2}(\varsigma(u))}{\partial x_{2}} d u\right)\left(\frac{\partial^{2} F_{2}(\varsigma(v))}{\partial x_{1} \partial x_{2}}\right)\right] \\
& \times\left[\int_{0}^{v} \exp \left(\int_{\theta}^{v} \frac{\partial F_{1}(\varsigma(u))}{\partial x_{1}} d u\right)\left(\frac{\partial F_{1}(\varsigma(\theta))}{\partial x_{2}}\right)\right. \\
& \left.\times \exp \left(\int_{0}^{\theta} \frac{\partial F_{2}(\varsigma(u))}{\partial x_{2}} d u\right) d \theta\right] d v \\
& =\int_{0}^{\tau_{0}} 2\left(\frac{b \beta \tilde{y}(v)}{a^{2}}-\frac{r}{k}\right) \exp \left(\int_{0}^{\tau_{0}}\left(r-\frac{\beta \tilde{y}(u)}{a}\right) d u\right) d v \\
& -\frac{\beta}{a}\left(\frac{\mu \beta}{a}-\eta\right) \int_{0}^{\tau_{0}} \exp \left(\int_{v}^{\tau_{0}}\left(r-\frac{\beta \tilde{y}(u)}{a}\right) d u\right) \\
& \times\left[\int_{0}^{v} \exp (-d(v-\theta)) \tilde{y}(\theta) \exp \left(\int_{0}^{\theta}\left(r-\frac{\beta \tilde{y}(u)}{a}\right) d u\right) d \theta\right] d v \\
& =\frac{2}{1-p_{1}}\left(\frac{b \beta p_{3}\left(1-\exp \left(-d \tau_{0}\right)\right)}{a^{2} d\left(1-\left(1-p_{2}\right) \exp \left(-d \tau_{0}\right)\right)}-\frac{r \tau_{0}}{k}\right) \\
& -\frac{\beta}{a}\left(\frac{\mu \beta}{a}-\eta\right) \int_{0}^{\tau_{0}} \exp \left(\int_{v}^{\tau_{0}}\left(r-\frac{\beta \tilde{y}(u)}{a}\right) d u\right) \\
& \times\left[\int_{0}^{v} \exp (-d(v-\theta)) \tilde{y}(\theta) \exp \left(\int_{0}^{\theta}\left(r-\frac{\beta \tilde{y}(u)}{a}\right) d u\right) d \theta\right] d v \text {. }
\end{aligned}
$$

Consider

$$
\begin{aligned}
\frac{b \beta p_{3}\left(1-\exp \left(-d \tau_{0}\right)\right)}{a^{2} d\left(1-\left(1-p_{2}\right) \exp \left(-d \tau_{0}\right)\right)}-\frac{r \tau_{0}}{k} & =\frac{b}{a}\left[r \tau_{0}-\ln \left(\frac{1}{1-p_{1}}\right)\right]-\frac{r \tau_{0}}{k} \\
& =\left(\frac{b}{a}-\frac{1}{k}\right) r \tau_{0}-\frac{b}{a} \ln \left(\frac{1}{1-p_{1}}\right) .
\end{aligned}
$$

It is not difficult to see from (73) and (74) that $\frac{\partial^{2} \Phi_{2}\left(\tau_{0}, X_{0}\right)}{\partial x_{2}^{2}}<0$ if the condition

$$
b k<a<\frac{\mu \beta}{\eta}
$$

is true. 
In addition, we have

$$
\begin{aligned}
& \frac{\partial \Theta_{1}}{\partial x_{1}}=1-p_{2}, \\
& \frac{\partial \Theta_{2}}{\partial x_{2}}=1-p_{1}, \\
& \frac{\partial \Theta_{1}}{\partial x_{2}}=\frac{\partial \Theta_{2}}{\partial x_{1}}=0 .
\end{aligned}
$$

Then we compute

$$
\begin{aligned}
d_{0}^{\prime} & \equiv 1-\left(\frac{\partial \Theta_{2}}{\partial x_{2}} \frac{\partial \Phi_{2}}{\partial x_{2}}\right)_{\left(\tau_{0}, X_{0}\right)} \\
& =1-\left(1-p_{1}\right) \exp \left(r \tau_{0}-\frac{\beta p_{3}\left(1-\exp \left(-d \tau_{0}\right)\right)}{a d\left(1-\left(1-p_{2}\right) \exp \left(-d \tau_{0}\right)\right)}\right)
\end{aligned}
$$

If $d_{0}^{\prime}=0$, then there is only one positive value of $\tau_{0}$ satisfying the equation

$$
r \tau_{0}-\frac{p_{3} \beta\left(1-\exp \left(-d \tau_{0}\right)\right)}{a d\left(1-\left(1-p_{2}\right) \exp \left(-d \tau_{0}\right)\right)}=\ln \left(\frac{1}{1-p_{1}}\right) .
$$

Furthermore, we obtain

$$
a_{0}^{\prime} \equiv 1-\left(\frac{\partial \Theta_{1}}{\partial x_{1}} \frac{\partial \Phi_{1}}{\partial x_{1}}\right)_{\left(\tau_{0}, X_{0}\right)}=1-\left(1-p_{2}\right) \exp \left(-d \tau_{0}\right)>0
$$

and

$$
\begin{aligned}
b_{0}^{\prime} \equiv & -\left(\frac{\partial \Theta_{1}}{\partial x_{1}} \frac{\partial \Phi_{1}}{\partial x_{2}}+\frac{\partial \Theta_{1}}{\partial x_{2}} \frac{\partial \Phi_{2}}{\partial x_{2}}\right)_{\left(\tau_{0}, X_{0}\right)} \\
= & -\left(1-p_{2}\right)\left(\frac{\mu \beta}{a}-\eta\right) \\
& \times \int_{0}^{\tau_{0}} \exp \left(-d\left(\tau_{0}-v\right)\right) \tilde{y}(v) \exp \left(\int_{0}^{v}\left(r-\frac{\beta \tilde{y}(u)}{a}\right) d u\right) d v .
\end{aligned}
$$

Since

$$
\int_{0}^{\tau_{0}} \exp \left(-d\left(\tau_{0}-v\right)\right) \tilde{y}(v) \exp \left(\int_{0}^{v}\left(r-\frac{\beta \tilde{y}(u)}{a}\right) d u\right) d v>0
$$

$b_{0}^{\prime}<0$ provided that the condition (75) holds. 
Now, we compute

$$
\begin{aligned}
B \equiv & -\frac{\partial^{2} \Theta_{2}}{\partial x_{1} \partial x_{2}}\left[\frac{\partial \Phi_{1}\left(\tau_{0}, X_{0}\right)}{\partial \tau}+\frac{\partial \Phi_{1}\left(\tau_{0}, X_{0}\right)}{\partial x_{1}} \frac{1}{a_{0}^{\prime}} \frac{\partial \Theta_{1}}{\partial x_{1}} \frac{\partial \Phi_{1}\left(\tau_{0}, X_{0}\right)}{\partial \tau}\right] \\
& \times\left(\frac{\partial \Phi_{2}\left(\tau_{0}, X_{0}\right)}{\partial x_{2}}\right) \\
& -\frac{\partial \Theta_{2}}{\partial x_{2}}\left[\frac{\partial^{2} \Phi_{2}\left(\tau_{0}, X_{0}\right)}{\partial \tau \partial x_{2}}+\frac{\partial^{2} \Phi_{2}\left(\tau_{0}, X_{0}\right)}{\partial x_{1} \partial x_{2}} \frac{1}{a_{0}^{\prime}} \frac{\partial \Theta_{1}}{\partial x_{1}} \frac{\partial \Phi_{1}\left(\tau_{0}, X_{0}\right)}{\partial \tau}\right] \\
= & -\frac{\partial \Theta_{2}}{\partial x_{2}}\left[\frac{\partial^{2} \Phi_{2}\left(\tau_{0}, X_{0}\right)}{\partial \tau \partial x_{2}}+\frac{\partial^{2} \Phi_{2}\left(\tau_{0}, X_{0}\right)}{\partial x_{1} \partial x_{2}} \frac{1}{a_{0}^{\prime}} \frac{\partial \Theta_{1}}{\partial x_{1}} \frac{\partial \Phi_{1}\left(\tau_{0}, X_{0}\right)}{\partial \tau}\right] \\
= & -\left(1-p_{1}\right)\left[\frac{1}{1-p_{1}}\left(r-\frac{\exp \left(-d \tau_{0}\right)}{a\left(1-\left(1-p_{2}\right) \exp \left(-d \tau_{0}\right)\right)}\right)\right. \\
& \left.+\frac{\partial^{2} \Phi_{2}\left(\tau_{0}, X_{0}\right)}{\partial x_{1} \partial x_{2}} \frac{1}{a_{0}^{\prime}} \frac{\partial \Theta_{1}}{\partial x_{1}} \frac{\partial \Phi_{1}\left(\tau_{0}, X_{0}\right)}{\partial \tau}\right] .
\end{aligned}
$$

Now, we know from the results obtained above that $\frac{\partial^{2} \Phi_{2}\left(\tau_{0}, X_{0}\right)}{\partial x_{1} \partial x_{2}}<0, a_{0}^{\prime}>0, \frac{\partial \Theta_{1}}{\partial x_{1}}>0$, and $\frac{\partial \Phi_{1}\left(\tau_{0}, X_{0}\right)}{\partial \tau}<0$. Next, we claim that $r-\frac{\beta p_{3} \exp \left(-d \tau_{0}\right)}{a\left(1-\left(1-p_{2}\right) \exp \left(-d \tau_{0}\right)\right)}>0$ so that one consequently obtains $B<0$. To verify the claim, we let

$$
\varphi(t)=r-\frac{\beta p_{3} \exp (-d t)}{a\left(1-\left(1-p_{2}\right) \exp \left(-d \tau_{0}\right)\right)} .
$$

Then we have

$$
\varphi^{\prime}(t)=\frac{d \beta p_{3} \exp (-d t)}{a\left(1-\left(1-p_{2}\right) \exp \left(-d \tau_{0}\right)\right)}>0 .
$$

Thus, $\varphi(t)$ is strictly increasing. In addition,

$$
\begin{aligned}
\int_{0}^{\tau_{0}} \varphi(t) d t & =r \tau_{0}-\frac{\beta p_{3}\left(1-\exp \left(-d \tau_{0}\right)\right)}{a d\left(1-\left(1-p_{2}\right) \exp \left(-d \tau_{0}\right)\right)} \\
& =\ln \left(\frac{1}{1-p_{1}}\right)>0
\end{aligned}
$$

Hence, we can conclude that $\varphi\left(\tau_{0}\right)>0$ according to the computations as shown above.

Finally, we calculate

$$
\begin{aligned}
C \equiv & -2 \frac{\partial^{2} \Theta_{2}}{\partial x_{1} \partial x_{2}}\left(-\frac{b_{0}^{\prime}}{a_{0}^{\prime}} \frac{\partial \Phi_{1}\left(\tau_{0}, X_{0}\right)}{\partial x_{1}}+\frac{\partial \Phi_{1}\left(\tau_{0}, X_{0}\right)}{\partial x_{2}}\right) \frac{\partial \Phi_{2}\left(\tau_{0}, X_{0}\right)}{\partial x_{2}} \\
& -\frac{\partial^{2} \Theta_{2}}{\partial x_{2}^{2}}\left(\frac{\partial \Phi_{2}\left(\tau_{0}, X_{0}\right)}{\partial x_{2}}\right)^{2}+2 \frac{\partial \Theta_{2}}{\partial x_{2}} \frac{b_{0}^{\prime}}{a_{0}^{\prime}} \frac{\partial^{2} \Phi_{2}\left(\tau_{0}, X_{0}\right)}{\partial x_{1} \partial x_{2}} \\
& -\frac{\partial \Theta_{2}}{\partial x_{2}} \frac{\partial^{2} \Phi_{2}\left(\tau_{0}, X_{0}\right)}{\partial x_{2}^{2}} \\
= & 2 \frac{\partial \Theta_{2}}{\partial x_{2}} \frac{b_{0}^{\prime}}{a_{0}^{\prime}} \frac{\partial^{2} \Phi_{2}\left(\tau_{0}, X_{0}\right)}{\partial x_{1} \partial x_{2}}-\frac{\partial \Theta_{2}}{\partial x_{2}} \frac{\partial^{2} \Phi_{2}\left(\tau_{0}, X_{0}\right)}{\partial x_{2}^{2}} \\
= & 2\left(1-p_{1}\right) \frac{b_{0}^{\prime}}{a_{0}^{\prime}} \frac{\partial^{2} \Phi_{2}\left(\tau_{0}, X_{0}\right)}{\partial x_{1} \partial x_{2}}-\left(1-p_{1}\right) \frac{\partial^{2} \Phi_{2}\left(\tau_{0}, X_{0}\right)}{\partial x_{2}^{2}}>0 .
\end{aligned}
$$


The above inequality is obtained via the fact that $a_{0}^{\prime}>0, \frac{\partial^{2} \Phi_{2}\left(\tau_{0}, X_{0}\right)}{\partial x_{1} \partial x_{2}}<0$ and $b_{0}^{\prime}<0$, $\frac{\partial^{2} \Phi_{2}\left(\tau_{0}, X_{0}\right)}{\partial x_{2}^{2}}<0$, which can be obtained using the condition (75). From the above notations, we have the following lemma.

Lemma 3.5 (see [59]) If $\left|1-a_{0}^{\prime}\right|<1$ and $d_{0}^{\prime}=0$, then the following results are obtained.

(a) If $B C \neq 0$, then one has a bifurcation. Specifically, one has a supercritical bifurcation of a nontrivial periodic solution of system (68) if $B C<0$ and a subcritical case if $B C>0$.

(b) If $B C=0$, then one has an undetermined case.

Letting $T_{\max } \equiv \tau_{0}$, where $\tau_{0}$ satisfies Eq. (78) and using Lemma 3.5 with the condition $B C<0$, one can conclude that system (7) has a supercritical bifurcation when $T>T_{\max }$ and $T$ is close to $T_{\max }$ as stated in the following theorem.

Theorem 3.3 System (7) has a positive periodic solution if $T>\tau_{0}$ and $T$ is close to $\tau_{0}$, where $\tau_{0}$ satisfies Eq. (78) and the nontrivial periodic solution is supercritical case via bifurcation, which means that the positive periodic solution is stable provided that the condition (75) holds.

\section{Numerical simulations}

The algorithm for solving impulsive differential equations is based on well-known numerical schemes [60-62] such as the spline approximation method, the $\theta$-method, the multistep method and the Runge-Kutta method. However, the adaptive step-size control should be incorporated as a part of such methods because it can handle an impulsive differential equation, considered as a stiff problem involving rapidly changing components together with slowly changing ones, quite well. Here, numerical simulations for system (7) are obtained using the built-in function ode15s [63] in MATLAB which is a variableorder, variable step size and multistep solver based on the backward differentiation formulas [64]. The entire time of investigation is partitioned into equal subintervals. Each of the subintervals has length $T$ (the impulsive period). The behavior of a solution for system (7) is characterized right before a pulse and immediately after the pulse by exploiting ode15s to numerically solve each subinterval of time. The information, evaluated at the jump point or the last time of the current subinterval, is used to compute the right limit of such a point. The right limit is then used as the initial condition of the next consecutive subinterval. The process is repeated in a similar manner for the rest subintervals and is terminated when we apply ode15s to perform numerical results for the last subinterval. The obtained numerical simulations in this section will be carried out to confirm our theoretical outcomes reported in Sect. 3. Studying the consequences of the impulsive effect to the dynamics of the impulsive prey-predator population model (7) will be done by the numerical experiments through adjusting the values of $p_{1}, p_{2}, p_{3}$ and $T$. Some parametric values for system $(7)$ are exactly taken from the literature $[23,30]$ but the others are appropriately estimated in order to satisfy the relevant conditions. The numerical simulations are as follows.

Figure 1 displays the simulation results of the impulsive system of Eq. (7) with the parameter values $r=3.1, k=2.067, \beta=1.083, a=1.031, b=0.001, \mu=0.85, d=0.3, \eta=0.01$. Figure 1 (a) shows the coexisting circumstance in which the prey and predator densities 

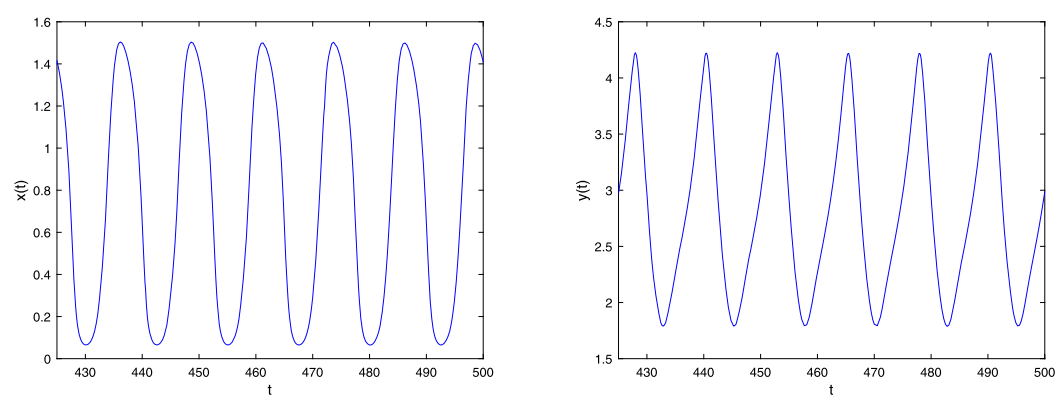

(a)
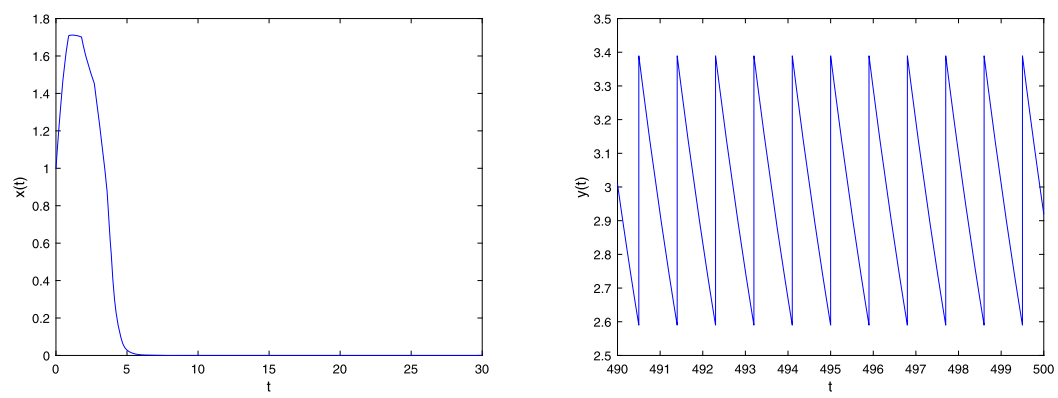

(b)
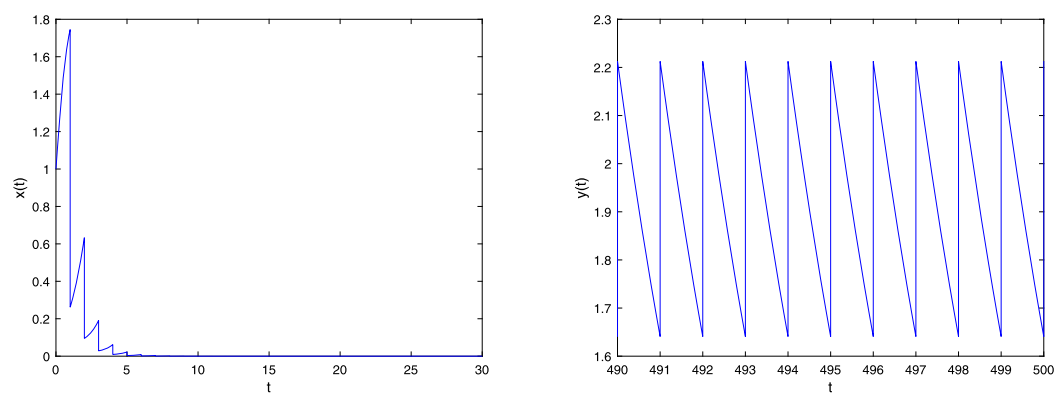

(c)

Figure 1 Time series simulations of the impulsive system in Eq. (7) using $r=3.1, k=2.067, \beta=1.083$, $a=1.031, b=0.001, \mu=0.85, d=0.3, \eta=0.01$ : (a) without taking any impulsive control strategy $\left(p_{1}=p_{2}=p_{3}=0\right)$ when $(x(0), y(0))=(0.5,0.5)$ and $T=13 ;(\mathbf{b})$ with only releasing the predators $\left(p_{1}=p_{2}=0, p_{3}=0.8\right)$ when $(x(0), y(0))=(1,1)$ and $T=0.8<T_{\max }=0.9 ;(\mathbf{c})$ with applying the IPM strategy $\left(p_{1}=0.85, p_{2}=0.2, p_{3}=0.9\right)$ when $(x(0), y(0))=(1,1)$ and $T=1<T_{\max }=1.335$

are the periodic oscillations starting at $(x(0), y(0))=(0.5,0.5)$. This is because the value of the parameters $p_{1}, p_{2}, p_{3}$ is all set to be zero, that is, any pest-management strategy is not yet applied to the system. The following numerical experiments confirm the result of Theorem 3.1 indicating that the solution of system (7) locally asymptotically converges to the prey-eradication periodic solution $(0, \tilde{y}(t))$ as $T<T_{\max }$. When we simulate the system with $(x(0), y(0))=(1,1)$ and $p_{1}=p_{2}=0, p_{3}=0.8$ (in other words, there is only a periodic releasing of the predators but without giving pesticide), then the solution trajectory tends toward the oscillatory solution $(0, \tilde{y}(t))$ when time passes long enough and $T=0.8<T_{\max }=0.9$. This phenomenon can be seen in Fig. 1(b). It can be observed from Fig. 1(c) that the predator density $y(t)$ oscillates in a stable periodic solution but the prey population $x(t)$ quickly reduces to zero. This prey-eradication periodic solution occurs 


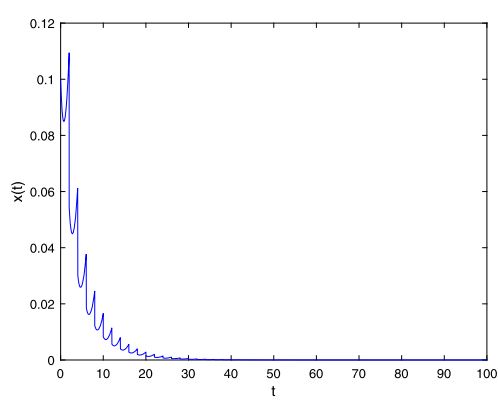

(a)

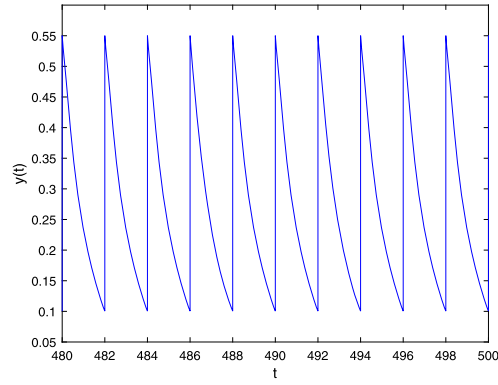

(b)

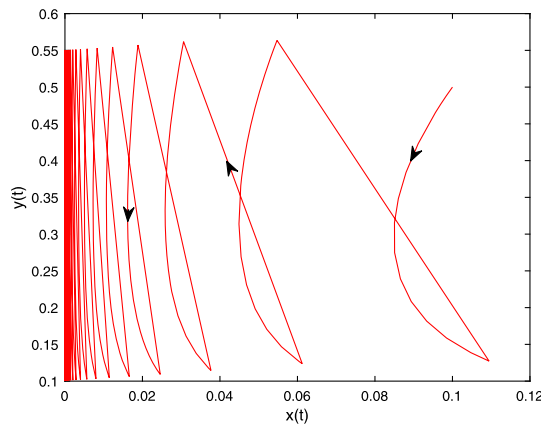

(c)

Figure 2 The numerical simulation of the impulsive system in Eq. (7) tends toward the periodically oscillatory solution $(0, \tilde{y}(t))$ using $r=0.8, k=0.5, \beta=0.7, a=0.3, b=0.05, \mu=0.85, d=0.85, \eta=0.1, p_{1}=p_{2}=p_{3}=0.5$ when $(x(0), y(0))=(0.1,0.5)$ and $T=2<T_{\max }=2.47$ : (a) the time series simulation of the prey density $x(t)$ approaching zero as $t$ is sufficiently large; (b) the time series simulation of the predator density $y(t)$ approaching a positive periodic solution as $t$ is large enough; (c) the trajectory simulation plotted on the xy-plane

when we set $p_{1}=0.85, p_{2}=0.2, p_{3}=0.9, x(0)=y(0)=1$ and $T=1<T_{\max }=1.335$ in the simulation. According to the used parameter values for the last two simulations, one can verify that condition (22) in Theorem 3.1 is satisfied.

In Fig. 2, the numerical simulation beginning with $(x(0), y(0))=(0.1,0.5)$ of the impulsive system of Eq. (7) locally asymptotically converges to the prey-eradication periodic solution $(0, \tilde{y}(t))$ when time sufficiently increases and $T=2<T_{\max }=2.47$. The parameter values used for this computation are $r=0.8, k=0.5, \beta=0.7, a=0.3, b=0.05, \mu=0.85, d=0.85$, $\eta=0.1, p_{1}=p_{2}=p_{3}=0.5$. With this IPM, the density of prey $x(t)$ converges asymptotically to zero but the density of predators $y(t)$ tends to a periodic oscillation of period $T=2$. This means that when $t$ is large enough, the prey population tends to extinction, however, the number of the predators changes in terms of the periodic oscillation. These obtained results are in good agreement with Theorem 3.1 since the condition (22) in Theorem 3.1 holds.

Next, we utilize the same parameter values and initial condition as used in the simulation for Fig. 2 except using $T=8>T_{\max }=2.47$ to simulate the numerical solution of the impulsive system (7). In consequence, the impulsive system with the use of this IPM is permanent as shown in Fig. 3. In other words, the prey and predator densities of the system are positively bounded as predicted in Theorem 3.2 because the condition (32) holds. 


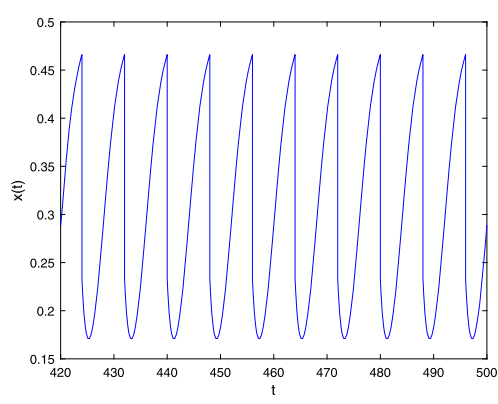

(a)

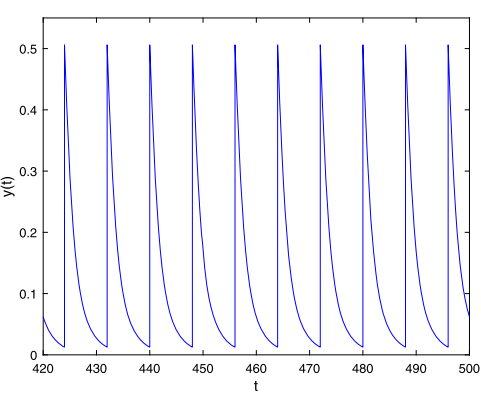

(b)

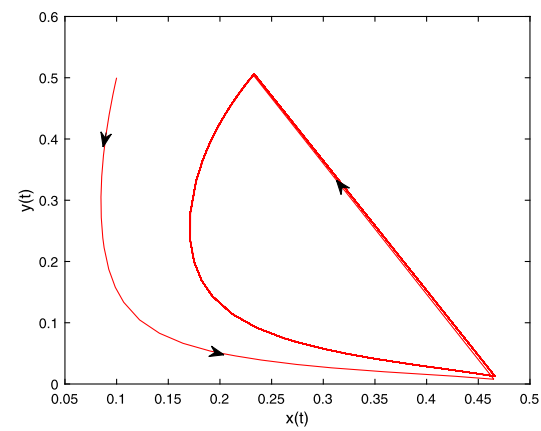

(c)

Figure 3 The numerical simulation shows the permanence of the impulsive system in Eq. (7). Here, the parameter values and the initial condition are exactly the same as used in Fig. 2 except $T=8>T_{\max }=2.47$ : (a) the bounded time series simulation of the prey density $x(t)$; (b) the bounded time series simulation of the predator density $y(t) ;(\mathbf{c})$ the trajectory simulation projected on the $x y$-plane

As a result, Figs. 3(a), (b) and (c) show the bounded time series of the prey density $x(t)$, the predator density $y(t)$ and their trajectory projected on the $x y$-plane, respectively.

In order to verify Theorem 3.3 applied for system (7), we must select the values of $T, k, \beta$, $a, b, \mu, \eta$ to satisfy all of the conditions in Theorem 3.3. Specifically, one can choose the impulsive period $T$ such that $T>T_{\max }$ and $T$ is close to $T_{\max }$. In the final simulation, we here use the same parameter values and initial condition as used in the simulation for Fig. 2 except using $T=2.7>T_{\max }=2.47$. The numerical solution plotted in Fig. 4 shows that the prey-eradication periodic solution $(0, \tilde{y}(t))$ becomes unstable and then the preys and predators can coexist, which this characterization is exhibited in terms of a sustained positive periodic oscillation when time passes and $T=2.7>T_{\max }=2.47$. This phenomenon allows the system to have a supercritical bifurcation, which can be used to control the magnitude of the prey population.

\section{Discussion and conclusions}

We have studied the dynamical behaviors of the mathematical impulsive model for the predator-prey system as proposed in Eq. (7). The impulsive system consists of the predator-prey model in which the generalized Holling type IV functional response and the anti-predator term are included and the impulsive control strategy concerning with giving pesticide and releasing of predators. Firstly, we have shown that system (7) has a positive solution which is uniformly ultimately bounded. Secondly, we have proved that the prey-eradication periodic solution $(0, \tilde{y}(t))$ is locally asymptotically stable provided 


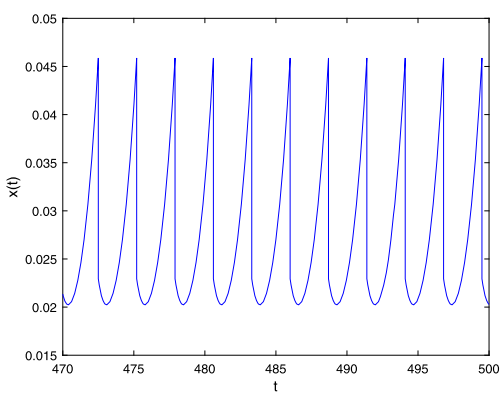

(a)

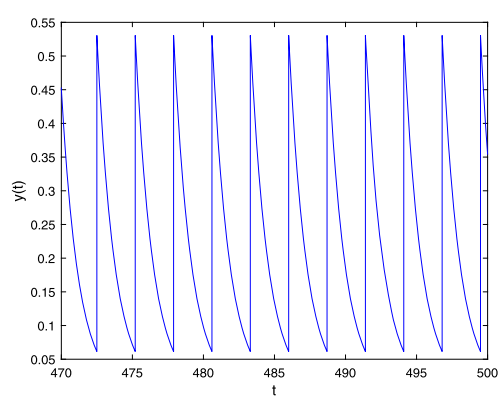

(b)

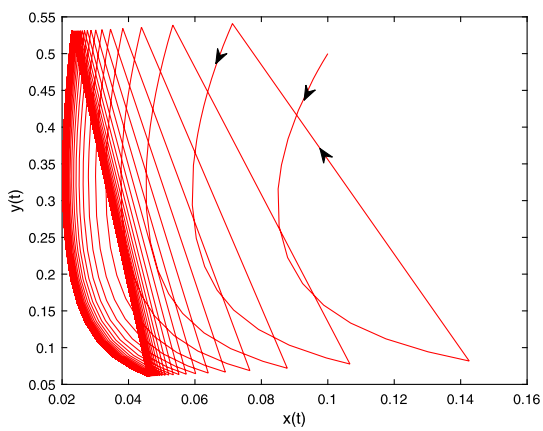

(c)

Figure 4 The numerical simulation demonstrates the occurrence of a stable positive periodic solution of the impulsive system in Eq. (7). Here, the parameter values and the initial condition are exactly the same as used in Fig. 2 except $T=2.7>T_{\max }=2.47$ : (a) the time series simulation of the prey density $x(t)$; $(\mathbf{b})$ the time series simulation of the predator density $y(t) ;(\mathbf{c})$ the trajectory simulation plotted on the $x y$-plane

that the impulsive period $T$ is less than the critical value $T_{\max }$, or equivalently the condition (22) holds. Practically, if the impulsive control strategy in system (7) is selected to push the preys to extinction, then we can determine the impulsive period $T$ based on the influences of spraying chemical pesticide and releasing natural predators such that $T<T_{\max }$. Of course, an absolute eradication of the prey population is not suitable for a real ecological system, so the effective prey-control practice should decrease the number of preys in the system to acceptable levels. In contrast, system (7) is permanent (i.e., the prey and predator populations are positively bounded) if $T>T_{\max }$. Profoundly, the system can lose the stability of the prey-eradication periodic solution and have a positive periodic solution when $T>T_{\max }$ and $T$ is close to $T_{\max }$. From the numerical experiments, we can observe that the narrower duration of $T$ results in the lower number of preys. Thirdly, system (7) can undergo either supercritical or subcritical bifurcations via choosing the values of the parameters in such a way that the sign of the discriminant $B C$ in Lemma 3.5 changes. Finally, changing the functional response, the anti-predator term or the IPM in system (7) could result in obtaining interesting and complicated dynamical behaviors of the preys and predators.

Acknowledgements

The authors are grateful to anonymous referees for the valuable comments, which have significantly improved this paper. 
Availability of data and materials

Not applicable.

\section{Competing interests}

The authors declare that they have no competing interests.

\section{Authors' contributions}

All authors worked together to produce the results, and they all read and approved the final manuscript.

\section{Publisher's Note}

Springer Nature remains neutral with regard to jurisdictional claims in published maps and institutional affiliations.

Received: 21 December 2020 Accepted: 25 February 2021 Published online: 09 March 2021

\section{References}

1. Chiu, K.-S., Li, T.: Oscillatory and periodic solutions of differential equations with piecewise constant generalized mixed arguments. Math. Nachr. 292(10), 2153-2164 (2019)

2. Li, T., Pintus, N., Viglialoro, G.: Properties of solutions to porous medium problems with different sources and boundary conditions. Z. Angew. Math. Phys. 70(3), 1-18 (2019)

3. Frassu, S., Viglialoro, G.: Boundedness for a fully parabolic Keller-Segel model with sublinear segregation and superlinear aggregation. Acta Appl. Math. 171(19), 1-20 (2021)

4. Liu, B., Zhang, Y., Chen, L.: The dynamical behaviors of a Lotka-Volterra predator-prey model concerning integrated pest management. Nonlinear Anal., Real World Appl. 6(2), 227-243 (2005)

5. Wang, Q., Dai, B., Chen, Y.: Multiple periodic solutions of an impulsive predator-prey model with Holling-type IV functional response. Math. Comput. Model. 49(9-10), 1829-1836 (2009)

6. Zeng, G., Wang, F., Nieto, J.J.: Complexity of a delayed predator-prey model with impulsive harvest and Holling type II functional response. Adv. Complex Syst. 11(01), 77-97 (2008)

7. Liu, M.: Optimal harvesting policy of a stochastic predator-prey model with time delay. Appl. Math. Lett. 48, 102-108 (2015)

8. Lotka, A.J.: Elements of physical biology. Sci. Prog. Twentieth Century (1919-1933) 21(82), 341-343 (1926)

9. Volterra, V.: Fluctuations in the abundance of a species considered mathematically. Nature 118, 558-560 (1926)

10. Tiwari, B., Raw, S., Mishra, P.: Qualitative analysis of a spatiotemporal prey-predator model with multiple Allee effect and schooling behaviour. Nonlinear Dyn. 102, 3013-3038 (2020)

11. Haldar, S., Khatua, A., Das, K., Kar, T.: Modeling and analysis of a predator-prey type eco-epidemic system with time delay. Model. Earth Syst. Environ. (2020). https://doi.org/10.1007/s40808-020-00893-9

12. Lai, L., Zhu, Z., Chen, F.: Stability and bifurcation in a predator-prey model with the additive Allee effect and the fear effect. Mathematics 8(8), 1280 (2020)

13. Kiss, K., Kovács, S.: Qualitative behavior of $n$-dimensional ratio-dependent predator-prey systems. Appl. Math. Comput. 199(2), 535-546 (2008)

14. Liu, X., Chen, L.: Complex dynamics of Holling type II Lotka-Volterra predator-prey system with impulsive perturbations on the predator. Chaos Solitons Fractals 16(2), 311-320 (2003)

15. Ma, Z., Chen, F., Wu, C., Chen, W.: Dynamic behaviors of a Lotka-Volterra predator-prey model incorporating a prey refuge and predator mutual interference. Appl. Math. Comput. 219(15), 7945-7953 (2013)

16. Zhao, X., Zeng, Z.: Stationary distribution and extinction of a stochastic ratio-dependent predator-prey system with stage structure for the predator. Phys. A, Stat. Mech. Appl. 545, 123310 (2020)

17. Yan, S., Guo, S.: Dynamics of a Lotka-Volterra competition-diffusion model with stage structure and spatial heterogeneity. Discrete Contin. Dyn. Syst., Ser. B 23(4), 1559 (2018)

18. Li, Y., Rafaqat, M., Zia, T.J., Ahmed, I., Jung, C.Y.: Flip and Neimark-Sacker bifurcations of a discrete time predator-prey model. IEEE Access 7, 123430-123435 (2019)

19. Raw, S., Tiwari, B., Mishra, P.: Dynamical complexities and pattern formation in an eco-epidemiological model with prey infection and harvesting. J. Appl. Math. Comput. 64, 17-52 (2020)

20. Prasad, K.D., Prasad, B.: Qualitative analysis of additional food provided predator-prey system with anti-predator behaviour in prey. Nonlinear Dyn. 96(3), 1765-1793 (2019)

21. Freedman, H.I., Wolkowicz, G.S.: Predator-prey systems with group defence: the paradox of enrichment revisited. Bull. Math. Biol. 48(5-6), 493-508 (1986)

22. Xiao, D., Ruan, S.: Global analysis in a predator-prey system with nonmonotonic functional response. SIAM J. Appl. Math. 61(4), 1445-1472 (2001)

23. Li, S.: Complex dynamical behaviors in a predator-prey system with generalized group defense and impulsive contro strategy. Discrete Dyn. Nat. Soc. 2013, Article ID 358930 (2013)

24. Tang, B.: Dynamics for a fractional-order predator-prey model with group defense. Sci. Rep. 10(1), 1-17 (2020)

25. Raw, S., Mishra, P., Kumar, R., Thakur, S.: Complex behavior of prey-predator system exhibiting group defense: a mathematical modeling study. Chaos Solitons Fractals 100, 74-90 (2017)

26. Sasmal, S.K., Takeuchi, Y.: Dynamics of a predator-prey system with fear and group defense. J. Math. Anal. Appl. 481(1), $123471(2020)$

27. Köhnke, M.C., Siekmann, I., Malchow, H.: Taxis-driven pattern formation in a predator-prey model with group defense. Ecol. Complex. 43, 100848 (2020)

28. Ives, A.R., Dobson, A.P. Antipredator behavior and the population dynamics of simple predator-prey systems. Am Nat. 130(3), 431-447 (1987)

29. Choh, Y., Ignacio, M., Sabelis, M.W., Janssen, A.: Predator-prey role reversals, juvenile experience and adult antipredator behaviour. Sci. Rep. 2, 728 (2012) 
30. Tang, B., Xiao, Y.: Bifurcation analysis of a predator-prey model with anti-predator behaviour. Chaos Solitons Fractals 70, 58-68 (2015)

31. Mortoja, S.G., Panja, P., Mondal, S.K.: Dynamics of a predator-prey model with stage-structure on both species and anti-predator behavior. Inform. Med. Unlock. 10, 50-57 (2018)

32. Liu, Q., Jiang, D., Hayat, T., Alsaedi, A.: Stationary distribution of a regime-switching predator-prey model with anti-predator behaviour and higher-order perturbations. Phys. A, Stat. Mech. Appl. 515, 199-210 (2019)

33. Raw, S., Mishra, P., Tiwari, B.: Mathematical study about a predator-prey model with anti-predator behavior. Int. J. Appl. Comput. Math. 6, 68 (2020)

34. Lima, S.L.: Stress and decision-making under the risk of predation: recent developments from behavioral, reproductive, and ecological perspectives. Adv. Study Behav. 27(8), 215-290 (1998)

35. Relyea, R.A.: How prey respond to combined predators: a review and an empirical test. Ecology 84(7), 1827-1839 (2003)

36. Janssen, A., Faraji, F., Van Der Hammen, T., Magalhães, S., Sabelis, M.W.: Interspecific infanticide deters predators. Ecol. Lett. 5(4), 490-494 (2002)

37. Saitō, Y.: Prey kills predator: counter-attack success of a spider mite against its specific phytoseiid predator. Exp. Appl. Acarol. 2(1), 47-62 (1986)

38. Sun, X., Li, Y., Xiao, Y.: A predator-prey model with prey population guided anti-predator behavior. Int. J. Bifurc. Chaos 27(07), 1750099 (2017)

39. Holling, C.S.: The functional response of predators to prey density and its role in mimicry and population regulation. Mem. Entomol. Soc. Can. 97(S45), 5-60 (1965)

40. Sarkar, K., Khajanchi, S., Chandra Mali, P., Nieto, J.J.: Rich dynamics of a predator-prey system with different kinds of functional responses. Complexity 2020, Article ID 4285294 (2020)

41. Köhnke, M.C., Siekmann, I., Seno, H., Malchow, H.: A type IV functional response with different shapes in a predator-prey model. J. Theor. Biol. 505, 110419 (2020)

42. Sokol, W., Howell, J.: Kinetics of phenol oxidation by washed cells. Biotechnol. Bioeng. 23(9), 2039-2049 (1981)

43. Huang, J.-C., Xiao, D.-M.: Analyses of bifurcations and stability in a predator-prey system with Holling type-IV functional response. Acta Math. Appl. Sin. 20(1), 167-178 (2004)

44. Parshad, R.D., Upadhyay, R.K., Mishra, S., Tiwari, S.K., Sharma, S.: On the explosive instability in a three-species food chain model with modified Holling type IV functional response. Math. Methods Appl. Sci. 40(16), 5707-5726 (2017)

45. Yousef, A., Rida, S., Gouda, Y.G., Zaki, A.: Dynamical behaviors of a fractional-order predator-prey model with Holling type iv functional response and its discretization. Int. J. Nonlinear Sci. Numer. Simul. 20(2), 125-136 (2019)

46. Roberts, M., Kao, R.: The dynamics of an infectious disease in a population with birth pulses. Math. Biosci. 149(1), 23-36 (1998)

47. Shulgin, B., Stone, L., Agur, Z.: Pulse vaccination strategy in the SIR epidemic model. Bull. Math. Biol. 60(6), 1123-1148 (1998)

48. Dees, H., Scott, T.: Medicaments for chemotherapeutic treatment of disease. Google Patents. US Patent App. $10 / 331,735(2003)$

49. Ballinger, G., Liu, X.: Permanence of population growth models with impulsive effects. Math. Comput. Model. 26(12), 59-72 (1997)

50. Vajrapatkul, A., Koonprasert, S., Sirisubtawee, S.: An application of the impulsive csoh model for managing squirrels in the coconut farm. Adv. Differ. Equ. 2019(1), 248 (2019)

51. Flint, M.L.: Integrated pest management for walnuts. University of California Statewide Integrated Pest Management Project, Division of Agriculture and Natural Resources, 2nd edn. Publication 3270, 3641 (1987)

52. Pei, Y., Chen, L., Zhang, Q., Li, C.: Extinction and permanence of one-prey multi-predators of Holling type II function response system with impulsive biological control. J. Theor. Biol. 235(4), 495-503 (2005)

53. Liu, J., Hu, J., Yuen, P.: Extinction and permanence of the predator-prey system with general functional response and impulsive control. Appl. Math. Model. 88, 55-67 (2020)

54. Li, W., Ji, J., Huang, L.: Global dynamic behavior of a predator-prey model under ratio-dependent state impulsive control. Appl. Math. Model. 77, 1842-1859 (2020)

55. Van Lenteren, J.: Integrated pest management in protected crops. Integrated pest management: principles and systems development (1995)

56. Liu, B., Zhi, Y., Chen, L.-S.: The dynamics of a predator-prey model with Ivlev's functional response concerning integrated pest management. Acta Math. Appl. Sin. 20(1), 133-146 (2004)

57. Lakshmikantham, V., Simeonov, P.S., et al.: Theory of Impulsive Differential Equations, vol. 6 (1989)

58. Baınov, D., Simeonov, P.: Impulsive Differential Equations: Periodic Solutions and Applications. Pitman Monographs and Surveys in Pure and Applied Mathematics, vol. 66. Longman, Harlow (1993)

59. Lakmeche, A.: Birfurcation of non trivial periodic solutions of impulsive differential equations arising chemotherapeutic treatment. Dyn. Contin. Discrete Impuls. Syst. 7, 265-287 (2000)

60. Randelovic, B., Stefanovic, L., Dankovic, B.: Numerical solution of impulsive differential equations. FACTA Univ. (NIS) Ser. Math. Info 15, 101-111 (2000)

61. Ran, X., Liu, M., Zhu, Q.: Numerical methods for impulsive differential equation. Math. Comput. Model. 48(1-2), 46-55 (2008)

62. Akça, H., Sarr, M., Covachev, V.: Numerical methods for solution of impulsive differential equations and stability analysis. Far East J. Math. Sci. 99(12), 1955 (2016)

63. Celaya, E.A., Aguirrezabala, J.A., Chatzipantelidis, P.: Implementation of an adaptive bdf2 formula and comparison with the Matlab ode15s. Proc. Comput. Sci. 29, 1014-1026 (2014)

64. Gear, C.W.: Numerical Initial Value Problems in Ordinary Differential Equations. Prentice-Hall Series in Automatic Computation (1971) 